TRANSACTIONS OF THE

AMERICAN MATHEMATICAL SOCIETY

Volume 362, Number 1, January 2010, Pages 289-318

S 0002-9947(09)04900-9

Article electronically published on August 4, 2009

\title{
DECAY FOR THE WAVE AND SCHRÖDINGER EVOLUTIONS ON MANIFOLDS WITH CONICAL ENDS, PART II
}

\author{
WILHELM SCHLAG, AVY SOFFER, AND WOLFGANG STAUBACH
}

\begin{abstract}
Let $\Omega \subset \mathbb{R}^{N}$ be a compact imbedded Riemannian manifold of dimension $d \geq 1$ and define the $(d+1)$-dimensional Riemannian manifold $\mathcal{M}:=\{(x, r(x) \omega): x \in \mathbb{R}, \omega \in \Omega\}$ with $r>0$ and smooth, and the natural metric $d s^{2}=\left(1+r^{\prime}(x)^{2}\right) d x^{2}+r^{2}(x) d s_{\Omega}^{2}$. We require that $\mathcal{M}$ has conical ends: $r(x)=|x|+O\left(x^{-1}\right)$ as $x \rightarrow \pm \infty$. The Hamiltonian flow on such manifolds always exhibits trapping. Dispersive estimates for the Schrödinger evolution $e^{i t \Delta_{\mathcal{M}}}$ and the wave evolution $e^{i t \sqrt{-\Delta_{\mathcal{M}}}}$ are obtained for data of the form $f(x, \omega)=Y_{n}(\omega) u(x)$, where $Y_{n}$ are eigenfunctions of $-\Delta_{\Omega}$ with eigenvalues $\mu_{n}^{2}$. In this paper we discuss all cases $d+n>1$. If $n \neq 0$ there is the following accelerated local decay estimate: with
\end{abstract}

$$
0<\sigma=\sqrt{2 \mu_{n}^{2}+(d-1)^{2} / 4}-\frac{d-1}{2}
$$

and all $t \geq 1$,

$$
\left\|w_{\sigma} e^{i t \Delta_{\mathcal{M}}} Y_{n} f\right\|_{L^{\infty}(\mathcal{M})} \leq C(n, \mathcal{M}, \sigma) t^{-\frac{d+1}{2}-\sigma}\left\|w_{\sigma}^{-1} f\right\|_{L^{1}(\mathcal{M})},
$$

where $w_{\sigma}(x)=\langle x\rangle^{-\sigma}$, and similarly for the wave evolution. Our method combines two main ingredients:

(A) A detailed scattering analysis of Schrödinger operators of the form $-\partial_{\xi}^{2}+\left(\nu^{2}-\frac{1}{4}\right)\langle\xi\rangle^{-2}+U(\xi)$ on the line where $U$ is real-valued and smooth with $U^{(\ell)}(\xi)=O\left(\xi^{-3-\ell}\right)$ for all $\ell \geq 0$ as $\xi \rightarrow \pm \infty$ and $\nu>0$. In particular, we introduce the notion of a zero energy resonance for this class and derive an asymptotic expansion of the Wronskian between the outgoing Jost solutions as the energy tends to zero. In particular, the division into Part I and Part II can be explained by the former being resonant at zero energy, where the present paper deals with the nonresonant case.

(B) Estimation of oscillatory integrals by (non)stationary phase.

\section{INTRODUCTION}

As in Part I, see [21, we consider the following class of manifolds $\mathcal{M}$ :

Definition 1.1. Let $\Omega \subset \mathbb{R}^{N}$ be an imbedded compact $d$-dimensional Riemannian manifold with metric $d s_{\Omega}^{2}$ and define the $(d+1)$-dimensional manifold

$$
\mathcal{M}:=\{(x, r(x) \omega) \mid x \in \mathbb{R}, \omega \in \Omega\}, \quad d s^{2}=r^{2}(x) d s_{\Omega}^{2}+\left(1+r^{\prime}(x)^{2}\right) d x^{2},
$$

Received by the editors December 14, 2007.

2000 Mathematics Subject Classification. Primary 35J10.

The first author was partly supported by the National Science Foundation grant DMS-0617854.

The second author was partly supported by the National Science Foundation grant DMS0501043 .

(C)2009 American Mathematical Society Reverts to public domain 28 years from publication 
where $r \in C^{\infty}(\mathbb{R})$ and $\inf _{x} r(x)>0$. We say that there is a conical end at the right (or left) if

$$
r(x)=|x|(1+h(x)), \quad h^{(k)}(x)=O\left(x^{-2-k}\right) \quad \forall k \geq 0
$$

as $x \rightarrow \infty(x \rightarrow-\infty)$.

Of course we can consider cones with arbitrary opening angles here but this adds nothing of substance. Examples of such manifolds are given by surfaces of revolution with $\Omega=S^{1}$ such as the one-sheeted hyperboloid. They have the property that the entire Hamiltonian flow on $\mathcal{M}$ is trapped on the set $\left(x_{0}, r\left(x_{0}\right) \Omega\right)$ when $r^{\prime}\left(x_{0}\right)=0$.

The main results of this paper are global-in-time dispersive estimates for the Schrödinger evolution $e^{i t \Delta_{\mathcal{M}}}$ and the wave evolution $e^{i t \sqrt{-\Delta_{\mathcal{M}}}}$, where $\Delta_{\mathcal{M}}$ denotes the Laplace-Beltrami operator on $\mathcal{M}$. These results should be contrasted to the large number of papers studying wave evolution on curved backgrounds; see, for example, [2], 4], 5], 9], [10], 11, [13, 14], 15], 17, [18, 22], 23], 24]. However, these references either consider the evolution on general manifolds for short times, or the global evolution on asymptotically flat perturbations of the Euclidean metric under a nontrapping condition. However, see the recent papers [16], 6] -8 , as well as the more classical paper [12 for semiclassical results close to a hyperbolic orbit of the Hamiltonian flow, and also [3] for local decay of the energy for the wave equation without any assumption on trapping.

The main point here is to carefully examine the long-time behavior for a class of examples that do exhibit trapping. In Part I we proved the case $d=1, n=0$, which is special (it can be viewed as an end-point case of the theory in Section 3 below).

In what follows, $\left\{Y_{n}, \mu_{n}\right\}_{n=0}^{\infty}$ denote the $L^{2}$-normalized eigenfunctions and eigenvalues, respectively, of $\Delta_{\Omega}$. In other words, $-\Delta_{\Omega} Y_{n}=\mu_{n}^{2} Y_{n}$ where $0=\mu_{0}^{2}<\mu_{1}^{2} \leq$ $\mu_{2}^{2} \leq \ldots$

Theorem 1.2. Let $\mathcal{M}$ be asymptotically conical at both ends in the sense of Definition 1.1 with $d \geq 1$ arbitrary. For each $d \geq 1, n \geq 0$, let

$$
\nu=\nu(d, n):=\sqrt{2 \mu_{n}^{2}+(d-1)^{2} / 4} .
$$

For each $n \geq 0$ and all $0 \leq \sigma \leq \nu(d, n)-\frac{d-1}{2}$, there exist constants $C(n, \mathcal{M}, \sigma)$ and $C_{1}(n, \mathcal{M}, \sigma)$ such that for all $t>0$,

$$
\begin{aligned}
\left\|w_{\sigma} e^{i t \Delta_{\mathcal{M}}} Y_{n} f\right\|_{L^{\infty}(\mathcal{M})} & \leq \frac{C(n, \mathcal{M}, \sigma)}{t^{\frac{d+1}{2}+\sigma}}\left\|\frac{f}{w_{\sigma}}\right\|_{L^{1}(\mathcal{M})}, \\
\left\|w_{\sigma} e^{ \pm i t \sqrt{-\Delta_{\mathcal{M}}}} Y_{n} f\right\|_{L^{\infty}(\mathcal{M})} & \leq \frac{C_{1}(n, \mathcal{M}, \sigma)}{t^{\frac{d}{2}+\sigma}}\left(\left\|\frac{f^{\prime}}{w_{\sigma}}\right\|_{L^{1}(\mathcal{M})}+\left\|\frac{f}{w_{\sigma}}\right\|_{L^{1}(\mathcal{M})}\right),
\end{aligned}
$$

provided $f=f(x)$ does not depend on $\omega$. Here $w_{\sigma}(x):=\langle x\rangle^{-\sigma}$ are weights on $\mathcal{M}$.

In our previous paper [21] we dealt with the case $d=1, n=0$ and proved (1.2) and (1.3) for that case. Needless to say, it is the analogue of the usual dispersive decay estimate for the Schrödinger and wave evolutions on $\mathbb{R}^{2}$. Clearly, the local decay given by $\sigma>0$ has no analogue in the Euclidean setting and it also has no meaning for $n=0$. To motivate it, one can try to rely on the geodesic flow on $\mathcal{M}$. As an example, take $\mathcal{M}$ to be the one-sheeted hyperboloid. It has a unique closed geodesic $\gamma_{0}$ at the neck and any other geodesic $\gamma$ that crosses $\gamma_{0}$ will pull away from it and never return. The analogue of $\mu_{n}=n$ would be the velocity of $\gamma$ 
and thus, the larger $n$, the faster $\gamma$ will pull away. This can serve to "explain" the improvement in terms of the power of $t$ for $\sigma>0$ in the following sense: imagine $f=f(x)$ to be a highly localized bump function centered around $\gamma_{0}$. Then the Schrödinger flow will (in phase space) mimic the geodesic flow on the cotangent bundle, at least for short times. By the "pulling away" logic we expect such data to disintegrate under the Schrödinger flow - and more strongly so as the angular momentum $n$ increases. Hence, as long as we only ask for the size of the solution close to $\gamma_{0}$ (this is the effect of the weight $w_{\sigma}$ ) we would expect to see very little of the wave left around the neck. Clearly, this is very much a negative curvature effect which should be contrasted to $\mathcal{M}=S^{d+1}$, for example.

However, this heuristic reasoning has to be taken with a grain of salt. First (and to the best of the authors' knowledge), it is not clear how to derive the exact power law above via a classical approximation. This is due to the fact that dispersive effects limit the accuracy of any classical approximation for long times (in more technical terms, this is the problem of constructing global parametrices by semi-classical methods). Second, note that Theorem 1.2 does not specify the behavior of $\mathcal{M}$ close to $x=0$. For example, Theorem 1.2 applies to a surface which is obtained as follows: cut the one-sheeted hyperboloid at the neck and glue the two pieces smoothly to a large sphere from which we have removed caps around the poles. The sphere of course has a continuum of stable closed geodesics. Nevertheless, due to the dispersion of the Schrödinger and wave flows the solution will spread into regions of $\mathcal{S}$ that exhibit the aforementioned instability of the geodesic flow typical of negatively curved surfaces. Theorem 1.2 states that over long times the power law is universal and does not see the local geometry. On a more technical level, let us mention that (1.2) and (1.3) are optimal with regard to both their respective $t^{-\frac{d+1}{2}-\sigma}$ and $t^{-\frac{d}{2}-\sigma}$ decay rates, as well as the polynomial weights $w_{\sigma}$ (in the sense that we cannot choose a smaller power) and the range of allowed $\sigma$.

A subtle point arises here, which is the size of the constants $C(n, \mathcal{S}, \sigma)$ and $C_{1}(n, \mathcal{S}, \sigma)$, especially with regard to their asymptotic behavior as $n \rightarrow \infty$. We do not address this asymptotic issue in $n$ at all in this paper. In fact, the methods of this paper were not designed with a view towards optimal constants but rather to exhibit the correct asymptotic behavior in $t$, and the constants $C(n, \mathcal{S}, \sigma), C_{1}(n, \mathcal{S}, \sigma)$ produced by our proof grow super-exponentially in $n$. A forthcoming paper will address the question of how our constants depend on $n$ by considering $\hbar=n^{-1}$ as a small semi-classical parameter. Hence, it is appropriate to view this paper as dealing with the intermediate regime of $n$, namely those that are not zero but not too large.

We believe that the analysis of the Laplacian $\Delta_{\mathcal{M}}$, which is carried out in Parts I and II, should be of independent interest and the Schrödinger and wave flow merely serve as an example where our asymptotic analysis applies. Note in particular that in Section 3 we develop the scattering theory of the class of Schrödinger operators on the line

$$
\mathcal{H}_{\nu}=-\partial_{\xi}^{2}+\left(\nu^{2}-\frac{1}{4}\right)\langle\xi\rangle^{-2}-U_{\nu}(\xi), \quad \frac{d^{\ell} U_{\nu}(\xi)}{d \xi^{\ell}}=O\left(\xi^{-3-\ell}\right)
$$

for all $\ell \geq 0$ as $\xi \rightarrow \pm \infty$ and $\nu>0$ (the $\nu$ is defined in Theorem 1.2). Section 3 is "abstract" in the sense that it does not draw on anything from the other sections. We obtain approximations to the Jost solutions of $\mathcal{H}_{\nu}$ as the energy $\lambda$ tends to 
zero and also find that their Wronskian is of the form $\lambda^{1-2 \nu}$ provided there is no zero energy resonance. By this we mean that the two subordinate solutions of the equation $\mathcal{H}_{\nu} f=0$ as $\xi \rightarrow \pm \infty$, respectively, do not form a globally subordinate solution on $\mathbb{R}$. See Definition 3.3 below. Although the notion of a zero energy resonance is standard for potentials that belong to $\left\{\langle\xi\rangle^{-1} V(\xi) \mid V \in L^{1}\right\}$, we are not aware of a reference where the conclusions of Section 3 are reached. This notion also helps to explain the difference between Part I and Part II: the former is resonant whereas the latter deals with the nonresonant case. This is in agreement with the fact that the Laplacian on $\mathbb{R}^{2}$ has a zero energy resonance, whereas on $\mathbb{R}^{n}$ with $n \geq 3$ it does not. The fact that Part $\mathrm{I}$ is resonant is due to the fact that the zero energy solutions are $u_{0}(\xi)=\sqrt{r(\xi)}$ and $u_{1}(\xi)=\sqrt{r(\xi)} \int_{0}^{\xi} \frac{d \eta}{r(\eta)}$. The subordinate one is $u_{0}$, which is global.

In the context of our conical manifolds we are able to settle the important resonant vs. nonresonant question by knowledge of the zero energy solutions of $\mathcal{H}_{\nu}$, which of course is equivalent to knowledge of the (spherical) harmonics of $\Delta_{\mathcal{M}}$. In fact, we shall see later on that for all $\nu>0$ the maximum principle allows us to conclude that we are in the nonresonant case (indeed, in the resonant case there would need to be a nonzero harmonic function on $\mathcal{M}$ that vanishes at both ends, which contradicts the maximum principle). As an example, for $d=1, n>0$ these functions are (with $\mu_{n}=n$ since $\Omega$ is isometric to $S^{1}$ for $d=1$ )

$$
\mathcal{H}_{1, n}\left(r^{\frac{1}{2}} e^{ \pm n y}\right)=0, \quad y(\xi)=\int_{0}^{\xi} \frac{d \eta}{r(\eta)} .
$$

Because $y$ is odd, it can agree with the larger branch only at one end, which places us in the nonresonant case.

Finally, let us remark that the methods of this paper cannot touch nonrotationally invariant perturbations of the metric on surfaces of revolution, let alone a non-symmetric example like three half-cones glued together smoothly (a "conical three-foil"). Another example would be two parallel planes joined by $k$ necks. While the case $k=1$ is essentially covered by Theorem 1.2 , the cases $k \geq 2$ are of course very different. It would be most interesting to find a way of approaching an analogue of Theorem 1.2 for manifolds which do not allow for separation of variables as we use here.

\section{The SETUP AND AN OVERVIEW OF ThE METHOD}

For the convenience of the reader, we reproduce some of the material from Section 2 of Part I. First, recall that the Laplace-Beltrami operator on $\mathcal{M}$, where the base $\Omega$ is of dimension $d \geq 1$, is

$$
\Delta_{\mathcal{M}}=\frac{1}{r^{d}(x) \sqrt{1+r^{\prime}(x)^{2}}} \partial_{x}\left(\frac{r^{d}(x)}{\sqrt{1+r^{\prime}(x)^{2}}} \partial_{x}\right)+\frac{1}{r^{2}(x)} \Delta_{\Omega} .
$$

In arclength parametrization,

$$
\xi(x)=\int_{0}^{x} \sqrt{1+r^{\prime}(y)^{2}} d y
$$


(2.1) reads

$$
\Delta_{\mathcal{M}}=\frac{1}{r^{d}(\xi)} \partial_{\xi}\left(r^{d}(\xi) \partial_{\xi}\right)+\frac{1}{r^{2}(\xi)} \Delta_{\Omega}
$$

where we have abused notation: $r(\xi)$ instead of $r(x(\xi))$. Setting $\rho(\xi):=\frac{d}{2} \frac{\dot{r}(\xi)}{r(\xi)}$ yields

$$
\Delta_{\mathcal{M}} y(\xi, \omega)=\partial_{\xi}^{2} y+2 \rho \partial_{\xi} y+\frac{1}{r^{2}} \Delta_{\Omega} y
$$

The first-order term in (2.3) is removed by setting

$$
y(\xi, \omega)=r(\xi)^{-\frac{d}{2}} u(\xi, \omega) .
$$

Then

$$
\Delta_{\mathcal{M}} y=\partial_{\xi}^{2} y+2 \rho \partial_{\xi} y+\frac{1}{r^{2}} \Delta_{\Omega} y=r^{-d / 2}\left[-\mathcal{H} u+\frac{1}{r^{2}} \Delta_{\Omega} u\right]
$$

with

$$
V_{1}(\xi):=\rho^{2}(\xi)+\dot{\rho}(\xi), \quad \mathcal{H}=-\partial_{\xi}^{2}+V_{1} .
$$

Note that the Schrödinger operator $\mathcal{H}$ can be factorized as

$$
\mathcal{H}=\mathcal{L}^{*} \mathcal{L}, \quad \mathcal{L}=-\frac{d}{d \xi}+\rho .
$$

In particular, $\mathcal{H}$ has no negative spectrum. Now specialize further to $u(\xi, \omega)=$ $Y_{n}(\omega) \phi(\xi)$. Then

$$
\mathcal{H} u-\frac{1}{r^{2}} \Delta_{\Omega} u=Y_{n} \mathcal{H}_{d, n} \phi, \quad \mathcal{H}_{d, n}=-\partial_{\xi}^{2}+V, \quad V(\xi):=V_{1}(\xi)+\frac{\mu_{n}^{2}}{r^{2}(\xi)} .
$$

The Schrödinger operator $\mathcal{H}_{d, n}$ is of fundamental importance to this paper. It has a smooth potential $V$ with the following asymptotic behavior. In Part I we proved that, see Lemma 2.2 and Corollary 2.3 of [21,

$$
\begin{aligned}
V(\xi)=V_{1}(\xi)+\frac{\mu_{n}^{2}}{r^{2}(\xi)} & =\left(2 \mu_{n}^{2}+d(d-2) / 4\right)\langle\xi\rangle^{-2}+O\left(\langle\xi\rangle^{-3}\right) \\
& =\left(\nu^{2}-\frac{1}{4}\right)\langle\xi\rangle^{-2}+O\left(\langle\xi\rangle^{-3}\right) .
\end{aligned}
$$

Here $\nu^{2}=2 \mu_{n}^{2}+(d-1)^{2} / 4$ is exactly as in Theorem 1.2 and the $O(\cdot)$ term behaves like a symbol, which means that

$$
\left|\partial_{\xi}^{\ell} O\left(\langle\xi\rangle^{-3}\right)\right| \leq C\langle\xi\rangle^{-3-\ell}, \quad \forall \ell \geq 0 .
$$

Note carefully that $d+n>1$ corresponds precisely to $\nu>0$ in $\mathcal{H}_{d, n}$. In terms of the Schrödinger evolution,

$$
e^{-i t \Delta_{\mathcal{M}}} Y_{n} f=r^{-\frac{d}{2}} Y_{n} e^{i t \mathcal{H}_{d, n}} r^{\frac{d}{2}} f \quad \forall f=f(\xi)
$$

and similarly for the wave equation. In particular, any estimate of the form

$$
\left\|w_{\sigma} e^{-i t \Delta_{\mathcal{M}}} Y_{n} f\right\|_{L^{\infty}(\mathcal{M})} \leq C t^{-\alpha}\left\|\frac{f}{w_{\sigma}}\right\|_{L^{1}(\mathcal{M})} \quad \forall t>0, f=f(\xi),
$$

with arbitrary $\alpha \geq 0$ and some constant $C$ that does not depend on $t$, is equivalent to one of the form

$$
\left\|r^{-\frac{d}{2}} w_{\sigma} e^{i t \mathcal{H}_{d, n}} r^{-\frac{d}{2}} u\right\|_{L^{\infty}(\mathbb{R})} \leq C^{\prime} t^{-\alpha}\left\|\frac{u}{w_{\sigma}}\right\|_{L^{1}(\mathbb{R})} \quad \forall t>0, u=u(\xi)
$$


with a possibly different constant $C^{\prime}$. Here we absorbed the weight from the volume element $d v_{\mathcal{M}}=r^{d} d \xi d v_{\Omega}$ arising in the $L^{1}(\mathcal{M})$ norm into the left-hand side of (2.8). An analogous reduction is of course valid for the wave evolution. As usual, the functional calculus applied to (2.8) yields

$$
e^{i t \mathcal{H}_{d, n}}=\int_{0}^{\infty} e^{i t \lambda} E(d \lambda),
$$

where $E(d \lambda)$ is the spectral resolution of $\mathcal{H}_{d, n}$. The point is that there is an "explicit expression" for $E(d \lambda)$ :

$$
\begin{aligned}
E\left(d \lambda^{2}\right)\left(\xi, \xi^{\prime}\right)=2 \lambda\{ & \operatorname{Im}\left[\frac{f_{+, \nu}(\xi, \lambda) f_{-, \nu}\left(\xi^{\prime}, \lambda\right)}{W_{\nu}(\lambda)}\right] \chi_{\left[\xi>\xi^{\prime}\right]} \\
& \left.+\operatorname{Im}\left[\frac{f_{-, \nu}(\xi, \lambda) f_{+, \nu}\left(\xi^{\prime}, \lambda\right)}{W_{\nu}(\lambda)}\right] \chi_{\left[\xi<\xi^{\prime}\right]}\right\} d \lambda
\end{aligned}
$$

where

$$
W_{\nu}(\lambda):=W\left(f_{-, \nu}(\cdot, \lambda), f_{+, \nu}(\cdot, \lambda)\right)=f_{+, \nu}^{\prime}(\cdot, \lambda) f_{-, \nu}(\cdot, \lambda)-f_{-, \nu}^{\prime}(\cdot, \lambda) f_{+, \nu}(\cdot, \lambda)
$$

is the Wronskian of the Jost solutions $f_{ \pm, \nu}(\cdot, \lambda)$ of the following ordinary differential equation:

$$
\begin{aligned}
\mathcal{H}_{d, n} f_{ \pm, \nu}(\xi, \lambda) & =-f_{ \pm, \nu}^{\prime \prime}(\xi, \lambda)+V(\xi) f_{ \pm, \nu}(\xi, \lambda)=\lambda^{2} f_{ \pm, \nu}(\xi, \lambda) \\
f_{ \pm, \nu}(\xi, \lambda) & \sim e^{ \pm i \lambda \xi} \quad \text { as } \xi \rightarrow \pm \infty
\end{aligned}
$$

provided $\lambda \neq 0$. It is a standard fact that these Jost solutions exist because of the decay $|V(\xi)| \lesssim\langle\xi\rangle^{-2}$. In fact, they are easily seen to exist provided the perturbing potential $V$ is in $L^{1}$; see [10]. Moreover, these Jost solutions are continuous in the energy $\lambda$ as $\lambda \rightarrow 0$ precisely when $\langle\xi\rangle V(\xi) \in L^{1}(\mathbb{R})$, which obviously fails here. On the other hand, it is common knowledge that the asymptotic form, i.e., the $t \rightarrow \infty$ decay law, of dispersive estimates like those in Theorem 1.2 crucially depends on the behavior of the spectral measure as $\lambda \rightarrow 0$. These two facts of course fit together well, as anything unusual like the accelerated local decay given by $\sigma>0$ must be reflected in the Jost solutions $f_{ \pm, \nu}(\cdot, \lambda)$ around $\lambda=0$. In fact, we show below that their Wronskian for $\lambda>0$ and with $\nu$ as in Theorem 1.2 satifies

$$
W\left(f_{+, \nu}(\cdot, \lambda), f_{-, \nu}(\cdot, \lambda)\right)=c_{\nu} \lambda^{1-2 \nu}\left(1+O\left(\lambda^{\varepsilon}\right)\right) \text { as } \lambda \rightarrow 0+.
$$

Here $\varepsilon>0$ is small depending on $\nu$; cf. Proposition 3.12 and Corollary 3.13 below. An intuitive way of viewing the leading-order behavior in (2.10) is as follows. From elementary quantum mechanics considerations we expect this leading order to be given by $\lambda e^{d_{A}(\lambda)}$ where $d_{A}(\lambda)$ is the Agmon distance between the turning points of $\mathcal{H}_{d, n}$ at energy $\lambda^{2}$ (actually, it turns out that one needs to ignore the $-\frac{1}{4} \xi^{-2}$ piece of $V$ for that purpose). Recall that the turning points $\xi_{1}<0<\xi_{2}$ are determined from the relation $\nu^{2}\left\langle\xi_{j}\right\rangle^{-2}=\lambda^{2}$ for $j=1,2$. The Agmon distance between $\xi_{1}$ and $\xi_{2}$ is then defined to be

$$
d_{A}(\lambda):=\int_{\xi_{1}}^{\xi_{2}} \sqrt{\nu^{2}\langle\xi\rangle^{-2}-\lambda^{2}} d \xi
$$

and thus

$$
d_{A}(\lambda)=2 \nu|\log \lambda|
$$

to leading order as $\lambda \rightarrow 0$. Finally, this exactly gives $\lambda e^{-2 \nu \log \lambda}=\lambda^{1-2 \nu}$ for the Wronskian as claimed; see (2.10). We caution the reader, though, that this heuristic 
via the Agmon distance only applies in the nonresonant case. See Section 3 , in particular Definition 3.3 .

In view of the preceding, the estimates (1.2) and (1.3) of Theorem 1.2 reduce to the following respective oscillatory integral estimates 1$]$ uniformly in $\xi>\xi^{\prime}$ (the case $\xi \leq \xi^{\prime}$ being analogous):

$$
\left|\int_{0}^{\infty} e^{i t \lambda^{2}} \lambda \operatorname{Im}\left[\frac{f_{+, \nu}(\xi, \lambda) f_{-, \nu}\left(\xi^{\prime}, \lambda\right)}{W_{\nu}(\lambda)}\right] d \lambda\right| \leq C(\nu, \mathcal{M}, \sigma)\left(\langle\xi\rangle\left\langle\xi^{\prime}\right\rangle\right)^{\frac{d}{2}+\sigma} t^{-\frac{d+1}{2}-\sigma},
$$

$$
\begin{aligned}
& \left|\int_{-\infty}^{\xi} \int_{0}^{\infty} e^{ \pm i t \lambda} \lambda \operatorname{Im}\left[\frac{f_{+, \nu}(\xi, \lambda) f_{-, \nu}\left(\xi^{\prime}, \lambda\right)}{W_{\nu}(\lambda)}\right] d \lambda\left(\langle\xi\rangle\left\langle\xi^{\prime}\right\rangle\right)^{-\frac{d}{2}-\sigma} \phi\left(\xi^{\prime}\right) d \xi^{\prime}\right| \\
& \leq C(\nu, \mathcal{M}, \sigma) t^{-\frac{d}{2}-\sigma} \int\left(\left|\phi^{\prime}(\eta)\right|+|\phi(\eta)|\right) d \eta
\end{aligned}
$$

It turns out that the $t^{-\nu}$ improvement over the usual $t^{-\frac{d+1}{2}}$ decay in (2.11) stems from the $\lambda^{-2 \nu}$ appearing in the Wronskian (2.10); indeed, we prove in this paper that the $\xi=\xi^{\prime}=0$ case of (2.11) reduces to the standard stationary phase type bound

$$
\left|\int_{0}^{\infty} e^{i t \lambda^{2}} \lambda^{1+2 \nu} \chi(\lambda) d \lambda\right| \leq C t^{-1-\nu}
$$

where $\chi$ is a smooth cut-off function to the interval $[0,1]$, say. Since $\frac{d+1}{2}+\sigma \leq$ $1+\nu$, this estimate implies the desired $t^{-\frac{d+1}{2}-\sigma}$ bound from (1.2). Note that this calculation also shows the optimality of the upper bound $\sigma \leq \nu-\frac{d-1}{2}$.

The reader should compare $W_{\nu}$ in (2.10) with the Wronskian for $n=0, d=1$ derived in 21]:

$$
W(\lambda)=2 \lambda\left(1+i c_{3}+i \frac{2}{\pi} \log \lambda\right)+O\left(\lambda^{\frac{3}{2}-\varepsilon}\right) \text { as } \lambda \rightarrow 0+.
$$

On a technical level, the logarithmic term in $\lambda$ makes the $n=0, d=1$ case of (2.11) somewhat harder to analyze than the cases $d+n>1$ (as already mentioned, $d+n=1$ is exactly $\nu=0$ ). Not surprisingly, in proving dispersive estimates for $-\Delta_{\mathbb{R}^{2}}+V$ one encounters similar logarithmic issues; see [19].

\section{The SCATtering THeORY of $\mathcal{H}_{\nu}, \nu>0$}

This section can and should be viewed as a separate entity, as it is kept completely general without any reference to the other sections. Our goal is to develop the scattering theory of the following class of operators $(\nu=0$ is treated in Part I, see [21]):

Definition 3.1. We define the class of operators

$$
\mathcal{H}_{\nu}:=-\partial_{\xi}^{2}+V(\xi)
$$

where $V \in C^{\infty}(\mathbb{R})$ is real-valued with the property that

$$
V(\xi)=\left(\nu^{2}-\frac{1}{4}\right) \xi^{-2}-U_{\nu}(\xi), \quad U_{\nu} \in C^{\infty}(\mathbb{R} \backslash\{0\})
$$

with $\nu>0, U_{\nu}$ real-valued and $U_{\nu}^{(\ell)}(\xi)=O\left(\xi^{-3-\ell}\right)$ for all $\ell \geq 0$ as $\xi \rightarrow \pm \infty$.

\footnotetext{
${ }^{1}$ We remark that the imaginary part in (2.11) is crucial.
} 
The goal here is to obtain representations of the Jost solutions $f_{ \pm, \nu}(\xi, \lambda)$ of $\mathcal{H}_{\nu}$ and their Wronskian $W_{\nu}(\lambda)$ especially as $\lambda \rightarrow 0$. Our main results concerning the class $\mathcal{H}_{\nu}$ are Proposition 3.12 and Corollary 3.13. We begin with certain bases of zero energy solutions $u_{0, \nu}^{ \pm}$and $u_{1, \nu}^{ \pm}$. All functions are smooth in $\xi$ where they are defined.

Lemma 3.2. There are solutions $u_{0, \nu}^{ \pm}$and $u_{1, \nu}^{ \pm}$of $\mathcal{H}_{\nu} f=0$ with the following properties:

$$
\begin{array}{ll}
u_{0, \nu}^{+}(\xi)=\xi^{\frac{1}{2}+\nu}\left(1+O\left(\xi^{-\alpha}\right)\right), & u_{1, \nu}^{+}(\xi)=\xi^{\frac{1}{2}-\nu}\left(1+O\left(\xi^{-1}\right)\right) \text { as } \xi \rightarrow \infty \\
u_{0, \nu}^{-}(\xi)=|\xi|^{\frac{1}{2}+\nu}\left(1+O\left(\xi^{-\alpha}\right)\right), & u_{1, \nu}^{-}(\xi)=|\xi|^{\frac{1}{2}-\nu}\left(1+O\left(\xi^{-1}\right)\right) \text { as } \xi \rightarrow-\infty
\end{array}
$$

The $O(\cdot)$ terms behave like symbols under differentiation in $\xi$ and $0<\alpha \leq \min (2 \nu, 1)$. Furthermore, the solutions $u_{1, \nu}^{ \pm}$are unique with the stated asymptotic behavior and

$$
W\left(u_{0, \nu}^{+}, u_{1, \nu}^{+}\right)=-2 \nu, \quad W\left(u_{0, \nu}^{-}, u_{1, \nu}^{-}\right)=2 \nu .
$$

Proof. We make the ansatz $y(\xi)=\xi^{\frac{1}{2}-\nu}(1+a(\xi))$ for $\xi>1$. Inserting this ansatz into the equation $\mathcal{H}_{\nu} y=0$ yields

$$
a(\xi)=-\int_{\xi}^{\infty} \int_{\xi}^{\zeta} \eta^{-1+2 \nu} d \eta U_{\nu}(\zeta) \zeta^{1-2 \nu}(1+a(\zeta)) d \zeta
$$

which is a Volterra equation of the form

$$
a(\xi)=\int_{\xi}^{\infty} K(\eta)(1+a(\eta)) d \eta, \quad K(\eta)=O\left(\eta^{-2}\right) \text { as } \eta \rightarrow \infty .
$$

The solution is of the form $a(\xi)=O\left(\xi^{-1}\right)$ where the $O(\cdot)$ is of symbol type. This gives the solution $u_{1, \nu}^{+}(\xi)$ with the desired properties. To find $u_{0, \nu}^{+}(\xi)$, we use the reduction ansatz, which yields (for some $\xi_{0}$ sufficiently large)

$$
u_{0, \nu}^{+}(\xi)=u_{1, \nu}^{+}(\xi) \int_{\xi_{0}}^{\xi}\left(u_{1, \nu}^{+}(\eta)\right)^{-2} d \eta=\xi^{\frac{1}{2}-\nu}(1+a(\xi)) \int_{\xi_{0}}^{\xi} \eta^{-1+2 \nu}(1+a(\eta))^{-2} d \eta
$$

If $\nu>\frac{1}{2}$, then

$$
u_{0, \nu}^{+}(\xi)=\xi^{\frac{1}{2}+\nu}\left(1+O\left(\xi^{-1}\right)\right) \text { as } \xi \rightarrow \infty
$$

whereas the range $0<\nu \leq \frac{1}{2}$ yields larger errors.

We can state a very important property in analogy with the case where $\langle\xi\rangle V \in$ $L^{1}$. In this paper we will only need the nonresonant case.

Definition 3.3. We say that $\mathcal{H}_{\nu}$ has a zero energy resonance iff

$$
W_{11}:=W\left(u_{1, \nu}^{+}, u_{1, \nu}^{-}\right)=0 \text {, }
$$

where $u_{1, \nu}^{ \pm}$are the unique solutions from Lemma 3.2. This is equivalent to the existence of a nonzero solution $f$ to $\mathcal{H}_{\nu} f=0$ so that $f(\xi)$ is asymptotic to $\xi^{\frac{1}{2}-\nu}$ as $\xi \rightarrow \infty$ and to $c|\xi|^{\frac{1}{2}-\nu}$ as $\xi \rightarrow-\infty$ with some constant $c \neq 0$.

We now perturb in energy to conclude the following. Let $\xi_{0}>0$ be fixed so that $u_{0, \nu}^{+}(\xi)>0$ for all $\xi \geq \xi_{0}$. 
Lemma 3.4. For any $\lambda \in \mathbb{R}$, define

$$
u_{0, \nu}^{+}(\xi, \lambda):=u_{0, \nu}^{+}(\xi)-\frac{\lambda^{2}}{2 \nu} \int_{\xi_{0}}^{\xi}\left[u_{1, \nu}^{+}(\xi) u_{0, \nu}^{+}(\eta)-u_{1, \nu}^{+}(\eta) u_{0, \nu}^{+}(\xi)\right] u_{0, \nu}^{+}(\eta, \lambda) d \eta .
$$

Then $\mathcal{H}_{\nu} u_{0, \nu}^{+}(\cdot, \lambda)=\lambda^{2} u_{0, \nu}^{+}(\cdot, \lambda)$.

Proof. Verify that

$$
(-2 \nu)^{-1}\left[u_{1, \nu}^{+}(\xi) u_{0, \nu}^{+}(\eta)-u_{1, \nu}^{+}(\eta) u_{0, \nu}^{+}(\xi)\right]
$$

is the backward Green function of $\mathcal{H}_{\nu}$.

Next, we extend $u_{0, \nu}^{+}(\cdot, \lambda)$ to a basis of solutions for $\mathcal{H}_{\nu} f=\lambda^{2} f$ for all small $\lambda>0$.

Corollary 3.5. Let $u_{0, \nu}^{+}(\cdot, \lambda)$ be defined as in (3.3). There exists a solution $u_{1, \nu}^{+}(\cdot, \lambda)$ of $\mathcal{H}_{\nu} f=\lambda^{2} f$ with

$$
W\left(u_{1, \nu}^{+}(\cdot, \lambda), u_{0, \nu}^{+}(\cdot, \lambda)\right)=1
$$

so that for $j=0,1$ and in the range $\xi_{0} \leq \xi \ll \lambda^{-1}$,

$$
u_{j, \nu}^{+}(\xi, \lambda)=u_{j, \nu}^{+}(\xi)\left(1+a_{j, \nu}^{+}(\xi, \lambda)\right),
$$

where

$$
\left|\partial_{\xi}^{\ell} \partial_{\lambda}^{k} a_{j, \nu}^{+}(\xi, \lambda)\right| \leq C_{k, \ell} \lambda^{2-k}\langle\xi\rangle^{2-\ell}
$$

for all $k, \ell \geq 0$ provided $\nu>1$. In the range $0<\nu \leq 1$ one has the weaker bounds

$$
\left|\partial_{\xi}^{\ell} \partial_{\lambda}^{k} a_{1, \nu}^{+}(\xi, \lambda)\right| \leq \begin{cases}C_{k, \ell} \lambda^{2 \nu-k}\langle\xi\rangle^{2 \nu-\ell} & \nu<1, \\ C_{k, \ell} \lambda^{2-k}\langle\xi\rangle^{2-\ell}|\log (\xi \lambda)| & \nu=1 .\end{cases}
$$

There is an analogous construction on $\xi \leq 0$.

Proof. With $u_{0, \nu}^{+}(\xi, \lambda)$ as in Lemma 3.4, write

$$
u_{0, \nu}^{+}(\xi, \lambda)=u_{0, \nu}^{+}(\xi) h(\xi, \lambda)
$$

for all $\xi>\xi_{0}$. Then

$$
\begin{aligned}
h(\xi, \lambda) & =1-\frac{\lambda^{2}}{2 \nu} \int_{\xi_{0}}^{\xi}\left[u_{1, \nu}^{+}(\eta) u_{0, \nu}^{+}(\eta)-\left(u_{0, \nu}^{+}\right)^{2}(\eta) \frac{u_{1, \nu}^{+}(\xi)}{u_{0, \nu}^{+}(\xi)}\right] h(\eta, \lambda) d \eta \\
& =1+\lambda^{2} \int_{\xi_{0}}^{\xi} K_{\nu}(\xi, \eta) h(\eta, \lambda) d \eta,
\end{aligned}
$$

where $\left|K_{\nu}(\xi, \eta)\right| \lesssim \eta$. Therefore, $h=1+O\left(\lambda^{2} \xi^{2}\right)$ as claimed. For the derivatives, use the symbol character of the $O(\cdot)$ terms from above. For $u_{1, \nu}^{+}$, we use the Wronskian condition:

$$
\partial_{\xi}\left[\frac{u_{1, \nu}^{+}(\xi, \lambda)}{u_{0, \nu}^{+}(\xi, \lambda)}\right]=\frac{-1}{\left(u_{0, \nu}^{+}(\xi, \lambda)\right)^{2}}
$$

and thus, with a sufficiently small constant $c$, we define

$$
u_{1, \nu}^{+}(\xi, \lambda):=u_{0, \nu}^{+}(\xi, \lambda) \int_{\xi}^{c \lambda^{-1}}\left(u_{0, \nu}^{+}(\eta, \lambda)\right)^{-2} d \eta .
$$

Inserting the expansion for $u_{0, \nu}^{+}(\eta, \lambda)$ into this expression finishes the proof. 
Next, we express the Jost solutions $f_{ \pm, \nu}(\xi, \lambda)$ of $\mathcal{H}_{\nu}$ in terms of the bases that we just constructed. Recall that the Jost solutions are defined as the unique solutions to the problem

$$
\mathcal{H}_{\nu} f_{ \pm, \nu}(\cdot, \lambda)=\lambda^{2} f_{ \pm, \nu}(\cdot, \lambda), \quad f_{ \pm, \nu}(\xi, \lambda) \sim e^{i \lambda \xi} \quad \text { as } \xi \rightarrow \pm \infty .
$$

Corollary 3.6. With $f_{ \pm, \nu}(\cdot, \lambda)$ being the Jost solutions of $\mathcal{H}_{\nu}$ with asymptotic behavior $e^{ \pm i \lambda \xi}$ as $\xi \rightarrow \pm \infty$, one has for any $\lambda \neq 0$,

$$
\begin{aligned}
& f_{+, \nu}(\xi, \lambda)=a_{+, \nu}(\lambda) u_{0, \nu}^{+}(\xi, \lambda)+b_{+, \nu}(\lambda) u_{1, \nu}^{+}(\xi, \lambda), \\
& f_{-, \nu}(\xi, \lambda)=a_{-, \nu}(\lambda) u_{0, \nu}^{-}(\xi, \lambda)+b_{-, \nu}(\lambda) u_{1, \nu}^{-}(\xi, \lambda),
\end{aligned}
$$

where $a_{ \pm, \nu}(\lambda)=-W\left(f_{ \pm, \nu}(\cdot, \lambda), u_{1, \nu}^{ \pm}(\cdot, \lambda)\right)$ and $b_{ \pm, \nu}(\lambda)=W\left(f_{ \pm, \nu}(\cdot, \lambda), u_{0, \nu}^{ \pm}(\cdot, \lambda)\right)$.

Proof. The Wronskian relations for $a_{ \pm, \nu}, b_{ \pm, \nu}$ follow immediately from (3.4).

To obtain an asymptotic expansion of $f_{ \pm, \nu}(\cdot, \lambda)$ for large $\xi$ we introduce

$$
\mathcal{H}_{0, \nu}=-\partial_{\xi}^{2}+\left(\nu^{2}-\frac{1}{4}\right) \xi^{-2}
$$

Lemma 3.7. For any $\lambda>0$ the problem

$$
\mathcal{H}_{0, \nu} f_{0, \nu}(\cdot, \lambda)=\lambda^{2} f_{0, \nu}(\cdot, \lambda), \quad f_{0, \nu}(\xi, \lambda) \sim e^{i \xi \lambda} \text { as } \xi \rightarrow \infty
$$

has a unique solution on $\xi>0$. It is given by

$$
f_{0, \nu}(\xi, \lambda)=\sqrt{\frac{\pi}{2}} e^{i(2 \nu+1) \pi / 4} \sqrt{\xi \lambda} H_{\nu}^{(+)}(\xi \lambda) .
$$

Here $H_{\nu}^{(+)}(z)=J_{\nu}(z)+i Y_{\nu}(z)$ is the Hankel function.

Proof. It is well known that the ordinary differential equation

$$
w^{\prime \prime}(z)+\left(\lambda^{2}-\frac{\nu^{2}-1 / 4}{z^{2}}\right) w(z)=0
$$

has a fundamental system $\sqrt{z} J_{\nu}(\lambda z), \sqrt{z} Y_{\nu}(\lambda z)$ or equivalently, $\sqrt{z} H_{\nu}^{(+)}(\lambda z)$, $\sqrt{z} H_{\nu}^{(-)}(\lambda z)$ (see [1]). Recall the asymptotics

$$
\begin{array}{ll}
H_{\nu}^{(+)}(x) \sim \sqrt{\frac{2}{\pi x}} e^{i\left(x-(2 \nu+1) \frac{\pi}{4}\right)} \quad & \text { as } x \rightarrow+\infty, \\
H_{\nu}^{(-)}(x) \sim \sqrt{\frac{2}{\pi x}} e^{-i\left(x-(2 \nu+1) \frac{\pi}{4}\right)} & \text { as } x \rightarrow+\infty .
\end{array}
$$

Thus, (3.9) is the unique solution so that $f_{0, \nu}(\xi, \lambda) \sim e^{i \xi \lambda}$, as claimed.

We now note the following: $\left\{f_{j}(x, \lambda)\right\}_{j=1,2}$ is a fundamental system of

$$
\mathcal{H}_{0, \nu} f=-f^{\prime \prime}+\frac{\nu^{2}-\frac{1}{4}}{x^{2}} f=f+\lambda U_{\nu}(x, \lambda) f \quad \text { on } \quad x>0,
$$

where $U_{\nu}(x, \lambda):=\lambda^{-3} U_{\nu}\left(\lambda^{-1} x\right)$ iff $\left\{f_{j}(\lambda \xi, \lambda)\right\}_{j=1,2}$ is a fundamental system of

$$
\mathcal{H}_{\nu} y(\cdot, \lambda)=\lambda^{2} y(\cdot, \lambda)
$$

We shall use the bounds

$$
\left|\partial_{x}^{\ell} \partial_{\lambda}^{k} U_{\nu}(x, \lambda)\right| \leq C_{k, \ell} x^{-3-\ell} \lambda^{-k} \quad \forall k, \ell \geq 0
$$

valid for all $x \geq \lambda$. 
Lemma 3.8. A fundamental system of (3.11) on $x \geq \frac{1}{2}$ is given by

$$
\phi_{1}(x, \lambda):=\sqrt{x} H_{\nu}^{(+)}(x)\left(1+\lambda b_{1}(x, \lambda)\right), \quad \phi_{2}(x, \lambda):=\overline{\phi_{1}(x, \lambda)}
$$

where 2

$$
\left|\partial_{x}^{\ell} \partial_{\lambda}^{k} b_{1}(x, \lambda)\right| \leq C_{k, \ell} \lambda^{-k} x^{-2-\ell} \quad \forall k, \ell \geq 0
$$

and all $x \geq \frac{1}{2}$.

Proof. Let $\phi_{0}(x):=\sqrt{x} H_{\nu}^{(+)}(x)$ and observe that $\phi_{0}(x)(1+\lambda b(x, \lambda))$ solves (3.11) iff

$$
b^{\prime \prime}(x, \lambda) \phi_{0}(x)+2 b^{\prime}(x, \lambda) \phi_{0}^{\prime}(x)=-U_{\nu}(x, \lambda) \phi_{0}(x)(1+\lambda b(x, \lambda))
$$

or

$$
b(x, \lambda)=-\int_{x}^{\infty} \phi_{0}^{2}(y)\left[\int_{x}^{y} \phi_{0}^{-2}(u) d u\right] U_{\nu}(y, \lambda)(1+\lambda b(y, \lambda)) d y .
$$

Define

$$
b_{0}(x, \lambda):=-\int_{x}^{\infty} \phi_{0}^{2}(y)\left[\int_{x}^{y} \phi_{0}^{-2}(u) d u\right] U_{\nu}(y, \lambda) d y .
$$

Note that $\phi_{0}$ never vanishes and satisfies the asymptotic expansion

$$
\phi_{0}(x)=c e^{i x}\left(1+O_{\mathbb{R}}\left(x^{-2}\right)+i O_{\mathbb{R}}\left(x^{-1}\right)\right)=e^{i x}\left(1+O_{\mathbb{C}}\left(x^{-1}\right)\right) \text { as } x \rightarrow \infty,
$$

where the $O_{\mathbb{R}}(\cdot)$ (or $O_{\mathbb{C}}(\cdot)$ ) terms are real-valued (or complex-valued) and behave like symbols under differentiation. In particular, integrating by parts shows that

$$
\sup _{y \geq x \geq \frac{1}{2}}\left|\phi_{0}^{2}(y)\left[\int_{x}^{y} \phi_{0}^{-2}(u) d u\right]\right| \lesssim 1,
$$

which implies that $\left|b_{0}(x, \lambda)\right| \lesssim x^{-2}$. The first derivative is given by

$$
\partial_{x} b_{0}(x, \lambda):=\int_{x}^{\infty} \phi_{0}^{2}(y) \phi_{0}^{-2}(x) U_{\nu}(y, \lambda) d y .
$$

Since

$$
\phi_{0}^{2}(y) \phi_{0}^{-2}(x)=e^{2 i(y-x)}\left(1+O_{\mathbb{C}}\left(x^{-1}\right)\right)\left(1+O_{\mathbb{C}}\left(y^{-1}\right)\right)
$$

we can integrate by parts in (3.17) to conclude that $\left|\partial_{x} b_{0}(x, \lambda)\right| \lesssim x^{-3}$. For the second derivative,

$$
\partial_{x}^{2} b_{0}(x, \lambda):=\int_{x}^{\infty} \partial_{x}\left[\phi_{0}^{2}(y) \phi_{0}^{-2}(x)\right] U_{\nu}(y, \lambda) d y-U_{\nu}(x, \lambda),
$$

we again integrate by parts using the identity (dropping the $\mathbb{C}$-subscript for the remainder of the proof)

$$
\begin{aligned}
\partial_{x}\left[\phi_{0}^{2}(y) \phi_{0}^{-2}(x)\right]=- & \partial_{y}\left[\phi_{0}^{2}(y) \phi_{0}^{-2}(x)\right] \\
& +e^{2 i(y-x)}\left[O\left(x^{-2}\right)\left(1+O\left(y^{-1}\right)\right)+O\left(y^{-2}\right)\left(1+O\left(x^{-1}\right)\right)\right] .
\end{aligned}
$$

\footnotetext{
${ }^{2}$ This can be strengthened to $\left|\operatorname{Im} \partial_{x}^{\ell} \partial_{\lambda}^{k} b_{1}(x, \lambda)\right| \leq C_{k, \ell} \lambda^{-k} x^{-2-\ell}$ and $\left|\operatorname{Re} \partial_{x}^{\ell} \partial_{\lambda}^{k} b_{1}(x, \lambda)\right| \leq$ $C_{k, \ell} \lambda^{-k} x^{-3-\ell}$.
} 
This yields

$$
\begin{aligned}
\partial_{x}^{2} b_{0}(x, \lambda) & :=\int_{x}^{\infty} \partial_{x}\left[\phi_{0}^{2}(y) \phi_{0}^{-2}(x)\right] U_{\nu}(y, \lambda) d y-U_{\nu}(x, \lambda) \\
& =\int_{x}^{\infty} \phi_{0}^{2}(y) \phi_{0}^{-2}(x) \partial_{y} U_{\nu}(y, \lambda) d y+O\left(x^{-4}\right) .
\end{aligned}
$$

Integrating by parts in the integral on line (3.19) we conclude that $\left|\partial_{x}^{2} b_{0}(x, \lambda)\right| \lesssim$ $x^{-4}$. Continuing in this fashion one proves that for all $\ell \geq 0$,

$$
\left|\partial_{x}^{\ell} b_{0}(x, \lambda)\right| \leq C_{\ell} x^{-2-\ell} .
$$

In view of (3.12), the $\lambda$-derivatives are treated in exactly the same way and we thus obtain the estimates

$$
\left|\partial_{x}^{\ell} \partial_{\lambda}^{k} b_{0}(x, \lambda)\right| \leq C_{\ell, k} x^{-2-\ell} \lambda^{-k}
$$

for all $k, \ell \geq 0$. These estimates transfer via (3.14) to $b(x, \lambda)$ because $\lambda$ is small. Indeed, first note that (3.14) has a solution via a contraction, say. Second, repeating the same arguments that lead to (3.20) but with (3.13) as bootstrap assumptions shows that we can get (3.13) back with the same constants (provided those are sufficiently large); the point is as follows: estimating $\partial_{x}^{\ell} b(x, \lambda)$ requires at most $\partial_{y}^{\ell-1} b(y, \lambda)$ inside the integral on the right-hand side of (3.14) (see (3.19) for the case $\ell=2)$. While $\partial_{\lambda}^{k} b(x, \lambda)$ with $k$ fixed can appear on both sides of (3.14), note that then we can use $\lambda$ small (with a smallness that does not depend on $k$ ) to solve for that derivative.

Next, we describe a basis of solutions for $\lambda \ll x \leq \frac{1}{2}$.

Lemma 3.9. A fundamental system of (3.11) on $\lambda \ll x \leq \frac{1}{2}$ is given by

$$
\psi_{1}(x, \lambda):=\sqrt{x} J_{\nu}(x)\left(1+\lambda c_{1}(x, \lambda)\right), \quad \psi_{2}(x, \lambda):=\sqrt{x} Y_{\nu}(x)\left(1+\lambda c_{2}(x, \lambda)\right),
$$

where for $j=1,2, c_{j}(x, \lambda)$ are real-valued and satisfy the bounds

$$
\left|\partial_{x}^{\ell} \partial_{\lambda}^{k} c_{j}(x, \lambda)\right| \leq C_{k, \ell} \lambda^{-k} x^{-1-\ell} \quad \forall k, \ell \geq 0
$$

and all $\lambda \ll x \leq \frac{1}{2}$.

Proof. As in the previous lemma, and since $Y_{\nu}(x)<0$ for all $0<x \leq \frac{1}{2}$ provided $\nu \geq 0$

$$
c_{2}(x, \lambda)=\int_{x}^{1} y Y_{\nu}^{2}(y) \int_{x}^{y} u^{-1} Y_{\nu}^{-2}(u) d u U_{\nu}(y, \lambda)\left(1+\lambda c_{2}(y, \lambda)\right) d y .
$$

Recall the asymptotic behavior, as $x \rightarrow 0+$ and with real constants $\alpha_{1, \nu}, \alpha_{2, \nu}$,

$$
J_{\nu}(x)=\alpha_{1, \nu} x^{\nu}\left(1+x \omega_{1}(x)\right), \quad Y_{\nu}(x)=\alpha_{2, \nu} x^{-\nu}\left(1+x \omega_{2}(x)\right),
$$

where the $\omega_{j}$ behave like symbols under differentiation: for all $\ell \geq 0$ and $j=1,2$,

$$
\left|\omega_{j}^{(\ell)}(x)\right| \leq C_{\ell} x^{-\ell}, \quad 0<x<\frac{1}{2} .
$$

First, let

$$
c_{2,0}(x, \lambda):=\int_{x}^{\frac{1}{2}} y Y_{\nu}^{2}(y) \int_{x}^{y} u^{-1} Y_{\nu}^{-2}(u) d u U_{\nu}(y, \lambda) d y
$$


Then for all $x>\lambda$,

$$
\begin{aligned}
\left|c_{2,0}(x, \lambda)\right| & \lesssim \int_{x}^{\frac{1}{2}} y^{1-2 \nu} \int_{x}^{y} u^{-1+2 \nu} d u\left|U_{\nu}(y, \lambda)\right| d y \lesssim \int_{x}^{\infty} y^{-2} d y \lesssim x^{-1}, \\
\left|\partial_{x} c_{2,0}(x, \lambda)\right| & \lesssim \int_{x}^{\frac{1}{2}} y Y_{\nu}^{2}(y) x^{-1} Y_{\nu}^{-2}(x)\left|U_{\nu}(y, \lambda)\right| d y \\
& \lesssim \int_{x}^{\frac{1}{2}} y^{1-2 \nu} x^{-1+2 \nu} y^{-3} d y \lesssim x^{-2} .
\end{aligned}
$$

Inductively, it now follows that

$$
\left|\partial_{x}^{\ell} \partial_{\lambda}^{k} c_{2,0}(x, \lambda)\right| \leq C_{k, \ell} \lambda^{-k} x^{-1-\ell} \quad \forall k, \ell \geq 0 .
$$

By a fixed-point argument, (3.22) has a solution $c_{2}(x, \lambda)$ for small $\lambda$ on $\lambda \leq x \leq \frac{1}{2}$ which satisfies (3.21) for all $k \geq 0$ and $\ell=0$. The same arguments that lead to (3.24) now yield (3.21) for all $\ell>0$ and $j=2$, settling the case of $\psi_{2}$.

The solution $\psi_{1}(x, \lambda)$ is given by, with a suitable constant $\gamma_{\nu} \neq 0$,

$$
\begin{aligned}
\psi_{1}(x, \lambda) & :=\gamma_{\nu}^{-1} \psi_{2}(x, \lambda) \int_{0}^{x} \psi_{2}^{-2}(y, \lambda) d y \\
& =\sqrt{x} Y_{\nu}(x)\left(1+\lambda c_{2}(x, \lambda)\right) \int_{0}^{x} y^{-1} Y_{\nu}(y)^{-2}\left(1+\lambda c_{2}(y, \lambda)\right)^{-2} d y
\end{aligned}
$$

for some sufficiently large constant $A$ which insures that

$$
\lambda c_{2} \ll 1 \text { on } A \lambda \leq x \leq \frac{1}{2} .
$$

Moreover, we set $c_{2}(x, \lambda):=0$ for all $0 \leq x \leq A \lambda$. Due to this fact, $\psi_{1}$ as defined in (3.25) solves (3.11) only on the interval $A \lambda \leq x \leq \frac{1}{2}$, which however is sufficient for our purposes. The constant $\gamma_{\nu} \neq 0$ is defined via the relation

$$
\gamma_{\nu} \sqrt{x} J_{\nu}(x)=\sqrt{x} Y_{\nu}(x) \int_{0}^{x} y^{-1} Y_{\nu}(y)^{-2} d y
$$

Hence, we see that

$$
\begin{aligned}
& \gamma_{\nu}^{-1} \sqrt{x} Y_{\nu}(x)\left(1+\lambda c_{2}(x, \lambda)\right) \int_{0}^{x} y^{-1} Y_{\nu}(y)^{-2}\left(1+\lambda c_{2}(y, \lambda)\right)^{-2} d y \\
& =\sqrt{x} J_{\nu}(x)\left(1+\lambda c_{2}(x, \lambda)\right)\left[1+\lambda Y_{\nu}(x) J_{\nu}^{-1}(x) \int_{0}^{x} y^{-1} Y_{\nu}(y)^{-2} O\left(c_{2}(y, \lambda)\right) d y\right] \\
& =\sqrt{x} J_{\nu}(x)\left(1+\lambda c_{2}(x, \lambda)\right)\left[1+\lambda O\left(x^{-2 \nu} x^{-1+2 \nu}\right)\right] \\
& =: \sqrt{x} J_{\nu}(x)\left(1+\lambda c_{1}(x, \lambda)\right)
\end{aligned}
$$

with $c_{1}$ inheriting the bounds (3.21) from $c_{2}$.

In what follows, $\beta_{\nu}:=\sqrt{\frac{\pi}{2}} e^{i(2 \nu+1) \pi / 4}$. We can now describe the Jost solutions of $\mathcal{H}_{\nu}$ in the region $1 \ll \xi \leq \lambda^{-1}$, which is needed for the matching described in Corollary 3.6 . 
Corollary 3.10. For $\lambda \neq 0$, let $f_{+, \nu}(\xi, \lambda)$ be the Jost solution satisfying (3.6). Then for all $1 \ll \xi \leq \lambda^{-1}$ there is the representation

$$
\begin{aligned}
f_{+, \nu}(\xi, \lambda)= & \beta_{\nu} \sqrt{\lambda \xi}\left[J_{\nu}(\lambda \xi)(1+O(\lambda))\left(1+O\left(\xi^{-1}\right)\right)+Y_{\nu}(\lambda \xi) O(\lambda)\left(1+O\left(\xi^{-1}\right)\right)\right] \\
& +i \beta_{\nu} \sqrt{\lambda \xi}\left[Y_{\nu}(\lambda \xi)(1+O(\lambda))\left(1+O\left(\xi^{-1}\right)\right)+J_{\nu}(\lambda \xi) O(\lambda)\left(1+O\left(\xi^{-1}\right)\right)\right]
\end{aligned}
$$

where each $O(\lambda)$ and $O\left(\xi^{-1}\right)$ is real-valued and behaves like a symbol under differentiation:

$$
\left|\partial_{\xi}^{\ell} \partial_{\lambda}^{k} O\left(\xi^{-1}\right)\right| \leq C_{k, \ell} \lambda^{-k} \xi^{-1-\ell}, \quad\left|\partial_{\xi}^{\ell} \partial_{\lambda}^{k} O(\lambda)\right| \leq C_{k, \ell} \lambda^{1-k} \xi^{-\ell} \quad \forall k, \ell \geq 0
$$

in the range $0<\lambda \ll 1,1 \ll \xi \leq \lambda^{-1}$.

Proof. In the rescaled picture, i.e., with $x=\lambda \xi$, the corresponding representation is given by

$$
\begin{aligned}
& \beta_{\nu} \sqrt{x}\left[J_{\nu}(x)(1+O(\lambda))\left(1+\lambda c_{1}(x, \lambda)\right)+Y_{\nu}(x) O(\lambda)\left(1+\lambda c_{2}(x, \lambda)\right)\right] \\
& +i \beta_{\nu} \sqrt{x}\left[Y_{\nu}(x)(1+O(\lambda))\left(1+\lambda c_{2}(x, \lambda)\right)+J_{\nu}(x) O(\lambda)\left(1+\lambda c_{1}(x, \lambda)\right)\right]
\end{aligned}
$$

as can be seen by matching the solutions of Lemma 3.9 to those of Lemma 3.8 at $x=1$. The $O(\lambda)$ have the claimed symbol behavior due to (3.13). Furthermore,

$$
\lambda c_{j}(\lambda \xi, \lambda)=O\left(\xi^{-1}\right)
$$

behaves under differentiation as claimed, see (3.21), and we are done.

The Wronskians appearing in Corollary 3.6 will be evaluated at $\xi=\lambda^{-1+\varepsilon}$, where $\varepsilon>0$ behaves like $\frac{1}{4 \nu}$. As a preliminary step, we note the following for $0<\lambda \ll 1$ :

Corollary 3.11. For $\lambda \neq 0$, let $f_{+, \nu}(\xi, \lambda)$ be the Jost solution of $\mathcal{H}_{\nu}$. Then for sufficiently small $\varepsilon>0$ and all $\lambda^{-1+\varepsilon} \leq \xi \leq \lambda^{-1}$ there is the representation

$$
f_{+, \nu}(\xi, \lambda)=\beta_{\nu} \sqrt{\lambda \xi} J_{\nu}(\lambda \xi)\left[1+O\left(\lambda^{\varepsilon}\right)\right]+i \beta_{\nu} \sqrt{\lambda \xi} Y_{\nu}(\lambda \xi)\left[1+O\left(\lambda^{1-\varepsilon}\right)\right],
$$

where $O\left(\lambda^{\varepsilon}\right)$ and $O\left(\lambda^{1-\varepsilon}\right)$ are real-valued and behave like symbols under differentiation:

$$
\left|\partial_{\xi}^{\ell} \partial_{\lambda}^{k} O\left(\lambda^{\varepsilon}\right)\right| \leq C_{k, \ell} \lambda^{\varepsilon-k} \xi^{-\ell}, \quad\left|\partial_{\xi}^{\ell} \partial_{\lambda}^{k} O\left(\lambda^{1-\varepsilon}\right)\right| \leq C_{k, \ell} \lambda^{1-\varepsilon-k} \xi^{-\ell} \quad \forall k, \ell \geq 0
$$

in the range $0<\lambda \ll 1, \lambda^{-1+\varepsilon} \leq \xi \leq \lambda^{-1}$.

Proof. Simply note that, for $\varepsilon \leq \frac{1}{4 \nu}$,

$$
\lambda \frac{Y_{\nu}(\lambda \xi)}{J_{\nu}(\lambda \xi)}=\lambda O\left((\lambda \xi)^{-2 \nu}\right)=O\left(\lambda^{\varepsilon}\right)
$$

in the specified range. Hence, (3.28) follows from (3.26). The behavior under differentiation is also clear from (3.27).

We now can compute the coefficients $a_{ \pm, \nu}(\lambda), b_{ \pm, \nu}(\lambda)$ and the Wronskian $W_{\nu}(\lambda)$ for small $\lambda$; see Corollary 3.6. Recall that $O\left(\lambda^{\sigma}\right)$ behaves like a symbol under differentiation if

$$
\partial_{\lambda}^{\ell} O\left(\lambda^{\sigma}\right)=O\left(\lambda^{\sigma-\ell}\right) \text { as } \lambda \rightarrow 0+
$$

for all $\ell \geq 0$. 
Proposition 3.12. Let $\beta_{\nu}$ be as above. With nonzero real constants $\alpha_{0, \nu}^{+}, \beta_{0, \nu}^{+}$, and some sufficiently small $\varepsilon>0$,

$$
\begin{aligned}
& a_{+, \nu}(\lambda)=\lambda^{\frac{1}{2}+\nu} \beta_{\nu}\left(\alpha_{0, \nu}^{+}+O\left(\lambda^{\varepsilon}\right)+i O\left(\lambda^{(1-2 \nu) \varepsilon}\right)\right), \\
& b_{+, \nu}(\lambda)=i \lambda^{\frac{1}{2}-\nu} \beta_{\nu}\left(\beta_{0, \nu}^{+}+O\left(\lambda^{\varepsilon}\right)+i O\left(\lambda^{(1+2 \nu) \varepsilon}\right)\right)
\end{aligned}
$$

as $\lambda \rightarrow 0+$ with real-valued $O(\cdot)$ which behave like symbols under differentiation in $\lambda$. The asymptotics as $\lambda \rightarrow 0$ - follows from that as $\lambda \rightarrow 0+$ via the relations $a_{+, \nu}(-\lambda)=\overline{a_{+, \nu}(\lambda)}, b_{+, \nu}(-\lambda)=\overline{b_{+, \nu}(\lambda)}$. Analogous expressions hold for $a_{-, \nu}$ and $b_{-, \nu}$.

Proof. Evaluating at $\xi=\lambda^{-1+\varepsilon}$, using (3.23) and Lemma 3.2

$$
\begin{aligned}
W\left(f_{+, \nu}(\cdot, \lambda), u_{1, \nu}^{+}(\cdot, \lambda)\right)= & \beta_{\nu} W\left(\sqrt{\lambda \xi} J_{\nu}(\lambda \xi)\left[1+O\left(\lambda^{\varepsilon}\right)\right], u_{1, \nu}^{+}(\xi)\left(1+O\left(\lambda^{2} \xi^{2}\right)\right)\right) \\
& +i \beta_{\nu} W\left(\sqrt{\lambda \xi} Y_{\nu}(\lambda \xi)\left[1+O\left(\lambda^{\varepsilon}\right)\right], u_{1, \nu}^{+}(\xi)\left(1+O\left(\lambda^{2} \xi^{2}\right)\right)\right) \\
= & \beta_{\nu} \alpha_{1, \nu} \lambda^{\frac{1}{2}+\nu} W\left(\xi^{\frac{1}{2}+\nu}\left[1+O\left(\lambda^{\varepsilon}\right)\right], \xi^{\frac{1}{2}-\nu}\left(1+O\left(\lambda^{\varepsilon}\right)\right)\right) \\
& +i \beta_{\nu} \alpha_{2, \nu} \lambda^{\frac{1}{2}-\nu} W\left(\xi^{\frac{1}{2}-\nu}\left[1+O\left(\lambda^{\varepsilon}\right)\right], \xi^{\frac{1}{2}-\nu}\left(1+O\left(\lambda^{\varepsilon}\right)\right)\right) \\
= & \beta_{\nu} \lambda^{\frac{1}{2}+\nu}\left[\alpha_{1, \nu}+O\left(\lambda^{\varepsilon}\right)+i O\left((\xi \lambda)^{-2 \nu} \lambda^{\varepsilon}\right)\right]
\end{aligned}
$$

with some constant $\tilde{\beta}_{\nu} \neq 0$. Next,

$$
\begin{aligned}
W\left(f_{+, \nu}(\cdot, \lambda), u_{0, \nu}^{+}(\cdot, \lambda)\right)= & \beta_{\nu} W\left(\sqrt{\lambda \xi} J_{\nu}(\lambda \xi)\left[1+O\left(\lambda^{\varepsilon}\right)\right], u_{0, \nu}^{+}(\xi)\left(1+O\left(\lambda^{2} \xi^{2}\right)\right)\right) \\
& +i \beta_{\nu} W\left(\sqrt{\lambda \xi} Y_{\nu}(\lambda \xi)\left[1+O\left(\lambda^{\varepsilon}\right)\right], u_{0, \nu}^{+}(\xi)\left(1+O\left(\lambda^{2} \xi^{2}\right)\right)\right) \\
= & \beta_{\nu} \alpha_{1, \nu} \lambda^{\frac{1}{2}+\nu} W\left(\xi^{\frac{1}{2}+\nu}\left[1+O\left(\lambda^{\varepsilon}\right)\right], \xi^{\frac{1}{2}+\nu}\left(1+O\left(\lambda^{\varepsilon}\right)\right)\right) \\
& +i \beta_{\nu} \alpha_{2, \nu} \lambda^{\frac{1}{2}-\nu} W\left(\xi^{\frac{1}{2}-\nu}\left[1+O\left(\lambda^{\varepsilon}\right)\right], \xi^{\frac{1}{2}+\nu}\left(1+O\left(\lambda^{\varepsilon}\right)\right)\right) \\
= & i \beta_{\nu} \lambda^{\frac{1}{2}-\nu}\left[\alpha_{2, \nu}+O\left(\lambda^{\varepsilon}\right)+i O\left((\xi \lambda)^{2 \nu} \lambda^{\varepsilon}\right)\right] .
\end{aligned}
$$

The proposition now follows by combining these calculations with Corollary 3.6 .

We can now describe the Wronskian $W_{\nu}(\lambda)=W\left(f_{-, \nu}(\cdot, \lambda), f_{+, \nu}(\cdot, \lambda)\right)$ in the nonresonant case:

Corollary 3.13. If $\mathcal{H}_{\nu}$ is nonresonant in the sense of Definition 3.3, then with some small $\varepsilon>0$ depending on $\nu$,

$$
\begin{aligned}
W_{\nu}(\lambda) & =b_{+, \nu}(\lambda) b_{-, \nu}(\lambda)\left(W_{11}+O\left(\lambda^{\varepsilon}\right)+i O\left(\lambda^{(1-2 \nu) \varepsilon}\right)\right) \\
& =i e^{i \nu \pi} \lambda^{1-2 \nu}\left(W_{0, \nu}+O_{\mathbb{C}}\left(\lambda^{\varepsilon}\right)\right) \text { as } \lambda \rightarrow 0+.
\end{aligned}
$$

Here $W_{0, \nu}$ is a nonzero real constant and $O_{\mathbb{C}}\left(\lambda^{\varepsilon}\right)$ is complex-valued and of symbol type. For $\lambda<0$, one has $W_{\nu}(-\lambda)=\overline{W_{\nu}(\lambda)}$.

Proof. In view of (3.7),

$$
\begin{aligned}
W_{\nu}(\lambda):= & W\left(f_{-, \nu}(\cdot, \lambda), f_{+, \nu}(\cdot, \lambda)\right) \\
= & a_{-, \nu}(\lambda) a_{+, \nu}(\lambda) W\left(u_{0, \nu}^{-}(\cdot, \lambda), u_{0, \nu}^{+}(\cdot, \lambda)\right) \\
& +a_{-, \nu}(\lambda) b_{+, \nu}(\lambda) W\left(u_{0, \nu}^{-}(\cdot, \lambda), u_{1, \nu}^{+}(\cdot, \lambda)\right) \\
+ & b_{-, \nu}(\lambda) a_{+, \nu}(\lambda) W\left(u_{1, \nu}^{-}(\cdot, \lambda), u_{0, \nu}^{+}(\cdot, \lambda)\right) \\
& +b_{-, \nu}(\lambda) b_{+, \nu}(\lambda) W\left(u_{1, \nu}^{-}(\cdot, \lambda), u_{1, \nu}^{+}(\cdot, \lambda)\right)
\end{aligned}
$$


for all $\lambda \neq 0$. From (3.5),

$$
W\left(u_{1, \nu}^{-}(\cdot, \lambda), u_{1, \nu}^{+}(\cdot, \lambda)\right)=W_{11}+O\left(\lambda^{\alpha}\right) \text { as } \lambda \rightarrow 0+,
$$

where $0<\alpha=\min (1,2 \nu)$ (with a logarithmic loss at $\nu=1$ ). By our nonresonant assumption, $W_{11} \neq 0$. Using the asymptotic expansions of Proposition 3.12 as well as setting

$$
W\left(u_{j, \nu}^{-}(\cdot, \lambda), u_{k, \nu}^{+}(\cdot, \lambda)\right)=O(1)
$$

for all $j+k<1$ finishes the proof.

\section{The SCATtering Theory of $\mathcal{H}_{d, n}, d+n>1$}

As explained in Section 2, we reduce the Laplacian on $\mathcal{M}$ to the Schrödinger operator

$$
\mathcal{H}_{d, n}=-\partial_{\xi}^{2}+\left(2 \mu_{n}^{2}+d(d-2) / 4\right)\langle\xi\rangle^{-2}+O\left(\langle\xi\rangle^{-3}\right),
$$

where the $O(\cdot)$ is of symbol type. This means that $\mathcal{H}_{d, n}=\mathcal{H}_{\nu}$ in the sense of Definition 3.1 with

$$
\nu=\sqrt{2 \mu_{n}^{2}+(d-1)^{2} / 4} .
$$

Note that $\nu>0$ unless $d=1, n=0$, which is not allowed here (this case was considered in Part I, see 21]). To be able to apply the results of Section 3, we need to verify the following:

Lemma 4.1. For any $d+n>1$ the operator $\mathcal{H}_{d, n}$ does not have a zero energy resonance in the sense of Definition 3.3 .

Proof. If there were a solution $u$ of $\mathcal{H}_{d, n} u=0$ with the property that $|u(\xi)| \lesssim|\xi|^{\frac{1}{2}-\nu}$ as $\xi \rightarrow \pm \infty$, then lifting this to $\mathcal{M}$ would yield a harmonic function decaying like $|\xi|^{1 / 2-2 \nu-d / 2}$ at both ends. But since $d \geq 1$, this would imply the existence of a nonzero harmonic function on $\mathcal{M}$ that vanishes at both ends (for all $\nu>0$ ). However, by the maximum principle such a harmonic function would need to vanish identically. This contradiction rules out a zero energy resonance of $\mathcal{H}_{d, n}$.

Thus, Proposition 3.12 and Corollary 3.13 apply to all $\mathcal{H}_{d, n}$ with $d+n>1$.

\section{The oscillatory integral estimates For $d+n>1$}

In this section we associate $\mathcal{H}_{d, n}$ with $\mathcal{H}_{\nu}$ as in the previous section. In fact, the estimates of this section do not use any other information about $\mathcal{H}_{d, n}$ than that furnished by Section 3 .

We begin with a corollary to Proposition 3.12. As already mentioned in Section 2, the importance of this corollary lies with the fact that the spectral resolution $E_{\nu}(d \lambda)\left(\xi, \xi^{\prime}\right)$ of $\mathcal{H}_{\nu}$ satisfies, for $\xi>\xi^{\prime}$,

$$
E_{\nu}\left(d \lambda^{2}\right)\left(\xi, \xi^{\prime}\right)=2 \lambda \operatorname{Im}\left[\frac{f_{+, \nu}(\xi, \lambda) f_{-, \nu}\left(\xi^{\prime}, \lambda\right)}{W_{\nu}(\lambda)}\right] d \lambda
$$

as an identity of Schwartz kernels. In this section we prove (2.11). We break this proof up into a small and a large energy piece. We also need to distinguish 
the oscillatory regime from the exponential regime in the Jost solutions $f_{ \pm, \nu}(\xi, \lambda)$ (the transition happens at $|\lambda \xi|=1$ ). This section will freely use the notation of Section 3 .

Corollary 5.1. For $0<\lambda \ll 1$ and any $\xi, \xi^{\prime} \in \mathbb{R}$,

$$
\begin{aligned}
& \operatorname{Im}\left[\frac{f_{+, \nu}(\xi, \lambda) f_{-, \nu}\left(\xi^{\prime}, \lambda\right)}{W_{\nu}(\lambda)}\right]=O\left(\lambda^{2 \nu}\right) u_{0, \nu}^{+}(\xi, \lambda) u_{1, \nu}^{-}\left(\xi^{\prime}, \lambda\right) \\
& \quad+O\left(\lambda^{2 \nu}\right) u_{1, \nu}^{+}(\xi, \lambda) u_{0, \nu}^{-}\left(\xi^{\prime}, \lambda\right)+O\left(\lambda^{2 \nu}\right) u_{0, \nu}^{+}(\xi, \lambda) u_{0, \nu}^{-}\left(\xi^{\prime}, \lambda\right) \\
& \quad+O\left(\lambda^{2 \nu}\right) u_{1, \nu}^{+}(\xi, \lambda) u_{1, \nu}^{-}\left(\xi^{\prime}, \lambda\right),
\end{aligned}
$$

where the $O(\cdot)$ are real-valued and behave like symbols under differentiation in $\lambda$.

Proof. By Corollary 3.6 one has

$$
\begin{aligned}
& \operatorname{Im}\left[\frac{f_{+, \nu}(\xi, \lambda) f_{-, \nu}\left(\xi^{\prime}, \lambda\right)}{W_{\nu}(\lambda)}\right] \\
& =\operatorname{Im}\left[\frac{\left(a_{+, \nu}(\lambda) u_{0, \nu}^{+}(\xi, \lambda)+b_{+, \nu}(\lambda) u_{1, \nu}^{+}(\xi, \lambda)\right)\left(a_{-, \nu}(\lambda) u_{0, \nu}^{-}\left(\xi^{\prime}, \lambda\right)+b_{-, \nu}(\lambda) u_{1, \nu}^{-}\left(\xi^{\prime}, \lambda\right)\right)}{W_{\nu}(\lambda)}\right] \\
& =\operatorname{Im}\left[\frac{a_{+, \nu} a_{-, \nu}(\lambda)}{W_{\nu}(\lambda)}\right] u_{0, \nu}^{+}(\xi, \lambda) u_{0, \nu}^{-}\left(\xi^{\prime}, \lambda\right)+\operatorname{Im}\left[\frac{b_{+, \nu} b_{-, \nu}(\lambda)}{W_{\nu}(\lambda)}\right] u_{1, \nu}^{+}(\xi, \lambda) u_{1, \nu}^{-}\left(\xi^{\prime}, \lambda\right) \\
& (5.2) \\
& \quad+\operatorname{Im}\left[\frac{a_{+, \nu} b_{-, \nu}(\lambda)}{W_{\nu}(\lambda)}\right] u_{0, \nu}^{+}(\xi, \lambda) u_{1, \nu}^{-}\left(\xi^{\prime}, \lambda\right)+\operatorname{Im}\left[\frac{a_{-, \nu} b_{+, \nu}(\lambda)}{W_{\nu}(\lambda)}\right] u_{0, \nu}^{-}(\xi, \lambda) u_{1, \nu}^{+}\left(\xi^{\prime}, \lambda\right) .
\end{aligned}
$$

One first verifies from (3.30) that

$$
W_{\nu}(\lambda)=b_{+, \nu}(\lambda) b_{-, \nu}(\lambda)\left[1+O\left(\lambda^{\varepsilon}\right)+i \lambda^{2 \nu}\left(\tau_{\nu}+O\left(\lambda^{\varepsilon}\right)+i O\left(\lambda^{(1-2 \nu) \varepsilon}\right)\right)\right]
$$

with real-valued $O(\cdot)$ terms and some real constant $\tau_{\nu}$. The four imaginary parts in (5.2) are now computed systematically from Proposition 3.12 and this expression. For example,

$$
\operatorname{Im}\left[\frac{b_{+, \nu} b_{-, \nu}(\lambda)}{W_{\nu}(\lambda)}\right]=\operatorname{Im}\left[1+O\left(\lambda^{\varepsilon}\right)+i \lambda^{2 \nu}\left(\tau_{\nu}+O\left(\lambda^{\varepsilon}+i O\left(\lambda^{(1-2 \nu) \varepsilon}\right)\right)\right]^{-1}=O\left(\lambda^{2 \nu}\right)\right.
$$

and

$$
\operatorname{Im}\left[\frac{a_{+, \nu} b_{-, \nu}(\lambda)}{W_{\nu}(\lambda)}\right]=\operatorname{Im} \frac{\lambda^{\frac{1}{2}+\nu}\left(\alpha_{0, \nu}^{+}+O\left(\lambda^{\varepsilon}\right)+i O\left(\lambda^{(1-2 \nu) \varepsilon}\right)\right)}{i \lambda^{\frac{1}{2}-\nu}\left(\beta_{0, \nu}^{+}+O\left(\lambda^{\varepsilon}\right)+i O\left(\lambda^{(1+2 \nu) \varepsilon}\right)\right)}=O\left(\lambda^{2 \nu}\right)
$$

as claimed. We leave the other two imaginary parts to the reader.

We now proceed to our first oscillatory integral estimate. Let $\chi$ be a smooth cutoff function to small energies, i.e., $\chi(\lambda)=1$ for small $|\lambda|$ and $\chi$ vanishes outside a small interval around zero. In addition, we introduce the smooth cutoff functions $\chi_{[|\xi \lambda|<1]}$ and $\chi_{[|\xi \lambda|>1]}$, which form a partition of unity adapted to these intervals. For the remainder of the paper, constants implicit in the $\lesssim$ notation of course do not depend on $t$. 
Lemma 5.2. For all $t>0$ and any $0 \leq \sigma \leq \nu-\frac{d-1}{2}$,

$$
\begin{aligned}
& \sup _{\xi, \xi^{\prime}}\left|\int_{0}^{\infty} e^{i t \lambda^{2}} \lambda \chi\left(\lambda ; \xi, \xi^{\prime}\right)\left(\langle\xi\rangle\left\langle\xi^{\prime}\right\rangle\right)^{-\frac{d}{2}-\sigma} \operatorname{Im}\left[\frac{f_{+, \nu}(\xi, \lambda) f_{-, \nu}\left(\xi^{\prime}, \lambda\right)}{W_{\nu}(\lambda)}\right] d \lambda\right| \lesssim t^{-\frac{d+1}{2}-\sigma}, \\
& (5.4) \\
& \sup _{\xi, \xi^{\prime}}\left|\int_{0}^{\infty} e^{ \pm i t \lambda} \lambda \chi\left(\lambda ; \xi, \xi^{\prime}\right)\left(\langle\xi\rangle\left\langle\xi^{\prime}\right\rangle\right)^{-\frac{d}{2}-\sigma} \operatorname{Im}\left[\frac{f_{+, \nu}(\xi, \lambda) f_{-, \nu}\left(\xi^{\prime}, \lambda\right)}{W_{\nu}(\lambda)}\right] d \lambda\right| \lesssim t^{-\frac{d}{2}-\sigma},
\end{aligned}
$$

where $\chi\left(\lambda ; \xi, \xi^{\prime}\right):=\chi(\lambda) \chi_{\left[|\xi \lambda|<1,\left|\xi^{\prime} \lambda\right|<1\right]}$.

Proof. We now write Corollary 5.1 schematically in the form

$$
\operatorname{Im}\left[\frac{f_{+, \nu}(\xi, \lambda) f_{-, \nu}\left(\xi^{\prime}, \lambda\right)}{W_{\nu}(\lambda)}\right]=O\left(\lambda^{2 \nu}\right) O\left(\left(\langle\xi\rangle\left\langle\xi^{\prime}\right\rangle\right)^{\frac{1}{2}+\nu}\right),
$$

where the second $O(\cdot)$ term is obtained from Corollary 3.5. Under differentiation in $\lambda$ the right-hand side behaves like a symbol. Thus, (5.3) reduces to the following stationary phase bound:

$$
\left|\int_{0}^{\infty} e^{i t \lambda^{2}} O\left(\lambda^{2 \nu+1}\right) \frac{\chi\left(\lambda ; \xi, \xi^{\prime}\right)}{\left(\langle\xi\rangle\left\langle\xi^{\prime}\right\rangle\right)^{\frac{d}{2}+\sigma}} O\left(\left(\langle\xi\rangle\left\langle\xi^{\prime}\right\rangle\right)^{\frac{1}{2}+\nu}\right) d \lambda\right| \lesssim t^{-\frac{d+1}{2}-\sigma} .
$$

Observe that

$$
O\left(\left(\langle\xi\rangle\left\langle\xi^{\prime}\right\rangle\right)^{\nu-\sigma-\frac{d-1}{2}}\right) \lambda^{2 \nu-2 \sigma-(d-1)}=O(1)
$$

on the support of the integrand (with symbol behavior under differentiation in $\lambda$ ). Hence, we conclude that it suffices to prove

$$
\left|\int_{0}^{\infty} e^{i t \lambda^{2}} \lambda^{d+2 \sigma} \omega\left(\lambda ; \xi, \xi^{\prime}\right) d \lambda\right| \lesssim t^{-\frac{d+1}{2}-\sigma}
$$

where for all $N \geq 1$,

$$
\sup _{\xi, \xi^{\prime}}\left|\partial_{\lambda}^{N} \omega\left(\lambda ; \xi, \xi^{\prime}\right)\right| \leq C_{\nu} \lambda^{-N}
$$

uniformly in $\xi, \xi^{\prime}$. However, this is a standard estimate and (5.3) follows.

The bound (5.4) is a consequence of the oscillatory integral estimate

$$
\left|\int_{0}^{\infty} e^{ \pm i t \lambda} \lambda^{d+2 \sigma} \omega\left(\lambda ; \xi, \xi^{\prime}\right) d \lambda\right| \lesssim \min \left(1, t^{-d-1-2 \sigma}\right)
$$

and the lemma follows.

Next, we consider the case $|\xi \lambda|>1$ and $\left|\xi^{\prime} \lambda\right|>1$. With the convention that $f_{ \pm}(\xi,-\lambda)=\overline{f_{ \pm}(\xi, \lambda)}$ we can remove the imaginary part from the resolvent and integrate $\lambda$ over the whole axis. Indeed, if $a\left(\lambda ; \xi, \xi^{\prime}\right)$ is an even function in $\lambda$, then

$$
\begin{aligned}
& \int_{0}^{\infty} e^{i t \lambda^{2}} \lambda \chi(\lambda) a\left(\lambda ; \xi, \xi^{\prime}\right) \operatorname{Im}\left[\frac{f_{+, \nu}(\xi, \lambda) f_{-, \nu}\left(\xi^{\prime}, \lambda\right)}{W_{\nu}(\lambda)}\right] d \lambda \\
& =\int_{0}^{\infty} e^{i t \lambda^{2}} \lambda \chi(\lambda) a\left(\lambda ; \xi, \xi^{\prime}\right)\left[\frac{f_{+, \nu}(\xi, \lambda) f_{-, \nu}\left(\xi^{\prime}, \lambda\right)}{W_{\nu}(\lambda)}-\frac{f_{+, \nu}(\xi,-\lambda) f_{-, \nu}\left(\xi^{\prime},-\lambda\right)}{W_{\nu}(-\lambda)}\right] d \lambda \\
& =\int_{-\infty}^{\infty} e^{i t \lambda^{2}} \lambda \chi(\lambda) a\left(\lambda ; \xi, \xi^{\prime}\right) \frac{f_{+, \nu}(\xi, \lambda) f_{-, \nu}\left(\xi^{\prime}, \lambda\right)}{W_{\nu}(\lambda)} d \lambda .
\end{aligned}
$$

We shall follow this convention henceforth. To estimate the oscillatory integrals, we shall repeatedly use the following version of the stationary phase; see Lemma 2 in [19] for the proof. 
Lemma 5.3. Let $\phi(0)=\phi^{\prime}(0)=0$ and $1 \leq \phi^{\prime \prime} \leq C$. Then

$$
\left|\int_{-\infty}^{\infty} e^{i t \phi(\lambda)} a(\lambda) d \lambda\right| \lesssim \delta^{2}\left\{\int \frac{|a(\lambda)|}{\delta^{2}+|\lambda|^{2}} d \lambda+\int_{|\lambda|>\delta} \frac{\left|a^{\prime}(\lambda)\right|}{|\lambda|} d \lambda\right\},
$$

where $\delta=t^{-1 / 2}$.

Before proceeding, let us note that

$$
\sup _{\xi, \xi^{\prime}}\left|\int_{-\infty}^{\infty} e^{i t \lambda^{2}} \lambda \chi(\lambda) \chi_{\left[|\xi \lambda|>1,\left|\xi^{\prime} \lambda\right|>1\right]}\left(\langle\xi\rangle\left\langle\xi^{\prime}\right\rangle\right)^{-\frac{d}{2}-\sigma} \frac{f_{+, \nu}(\xi, \lambda) f_{-, \nu}\left(\xi^{\prime}, \lambda\right)}{W_{\nu}(\lambda)} d \lambda\right| \lesssim 1
$$

due to the fact that

$$
\sup _{\xi, \xi^{\prime}}|\lambda|\left|\frac{f_{+, \nu}(\xi, \lambda) f_{-, \nu}\left(\xi^{\prime}, \lambda\right)}{W_{\nu}(\lambda)}\right| \lesssim 1
$$

see Section 3, Hence, in all small energy oscillatory integrals it suffices to consider $t>1$. The same comment applies of course to the wave equation.

Using Lemma 5.3 we can prove the following:

Lemma 5.4. For all $t \geq 1$ and $0 \leq \sigma \leq \nu-\frac{d-1}{2}$,

$$
\begin{gathered}
\sup _{\xi>0>\xi^{\prime}}\left|\int_{-\infty}^{\infty} e^{i t \lambda^{2}} \lambda \chi(\lambda) \chi_{\left[|\xi \lambda|>1,\left|\xi^{\prime} \lambda\right|>1\right]}\left(\langle\xi\rangle\left\langle\xi^{\prime}\right\rangle\right)^{-\frac{d}{2}-\sigma} \frac{f_{+, \nu}(\xi, \lambda) f_{-, \nu}\left(\xi^{\prime}, \lambda\right)}{W_{\nu}(\lambda)} d \lambda\right| \\
\lesssim t^{-\frac{d+1}{2}-\sigma}, \\
\sup _{\xi>0>\xi^{\prime}}\left|\int_{-\infty}^{\infty} e^{ \pm i t \lambda} \lambda \chi(\lambda) \chi_{\left[|\xi \lambda|>1,\left|\xi^{\prime} \lambda\right|>1\right]}\left(\langle\xi\rangle\left\langle\xi^{\prime}\right\rangle\right)^{-\frac{d}{2}-\sigma} \frac{f_{+, \nu}(\xi, \lambda) f_{-, \nu}\left(\xi^{\prime}, \lambda\right)}{W_{\nu}(\lambda)} d \lambda\right| \\
\lesssim t^{-\frac{d}{2}-\sigma} .
\end{gathered}
$$

Proof. Writing

$$
f_{+, \nu}(\xi, \lambda)=e^{i \xi \lambda} m_{+, \nu}(\xi, \lambda), \quad f_{-, \nu}(\xi, \lambda)=e^{-i \xi \lambda} m_{-, \nu}(\xi, \lambda),
$$

one infers from Lemma 3.8 that

$$
\left|m_{+, \nu}(\xi, \lambda)-1\right| \lesssim \lambda^{-1} \xi^{-1}, \quad\left|\partial_{\lambda} m_{+, \nu}(\xi, \lambda)\right| \lesssim \lambda^{-2} \xi^{-1} \lesssim \lambda^{-1} .
$$

Indeed, simply set

$$
m_{+, \nu}(\xi, \lambda):=\left(1+\lambda b_{1}(\lambda \xi, \lambda)\right)\left(1+O\left(\lambda^{-1} \xi^{-1}\right)\right),
$$

where $b_{1}$ is from that lemma. Next, we express (5.6) in the form

$$
\left|\int_{-\infty}^{\infty} e^{i t \phi\left(\lambda ; \xi, \xi^{\prime}\right)} a_{\nu}\left(\lambda ; \xi, \xi^{\prime}\right) d \lambda\right| \lesssim t^{-\frac{d+1}{2}-\sigma}
$$

where $\xi>0>\xi^{\prime}$ are fixed, $\phi\left(\lambda ; \xi, \xi^{\prime}\right):=\lambda^{2}+\frac{\lambda}{t}\left(\xi-\xi^{\prime}\right)$, and

$$
a_{\nu}\left(\lambda ; \xi, \xi^{\prime}\right):=\lambda \chi(\lambda) \chi_{\left[|\xi \lambda|>1,\left|\xi^{\prime} \lambda\right|>1\right]}\left(\langle\xi\rangle\left\langle\xi^{\prime}\right\rangle\right)^{-\frac{d}{2}-\sigma} \frac{m_{+, \nu}(\xi, \lambda) m_{-, \nu}\left(\xi^{\prime}, \lambda\right)}{W_{\nu}(\lambda)} .
$$

Denote the critical point of $\phi$ by $\lambda_{0}:=-\frac{\xi-\xi^{\prime}}{2 t}$. By Proposition 3.12, for small $|\lambda|$,

$$
\left|\frac{\lambda}{W_{\nu}(\lambda)}\right| \lesssim \lambda^{2 \nu}, \quad\left|\left(\frac{\lambda}{W_{\nu}(\lambda)}\right)^{\prime}\right| \lesssim \lambda^{2 \nu-1}
$$


Hence,

$$
\begin{aligned}
\left|a_{\nu}\left(\lambda ; \xi, \xi^{\prime}\right)\right| & \lesssim \lambda^{2 \nu}\left(\langle\xi\rangle\left\langle\xi^{\prime}\right\rangle\right)^{-\frac{d}{2}-\sigma} \chi(\lambda) \chi_{\left[|\xi \lambda|>1,\left|\xi^{\prime} \lambda\right|>1\right]}, \\
\left|\partial_{\lambda}^{\ell} a_{\nu}\left(\lambda ; \xi, \xi^{\prime}\right)\right| & \lesssim \lambda^{2 \nu-\ell}\left(\langle\xi\rangle\left\langle\xi^{\prime}\right\rangle\right)^{-\frac{d}{2}-\sigma} \chi(\lambda) \chi_{\left[|\xi \lambda|>1,\left|\xi^{\prime} \lambda\right|>1\right]}
\end{aligned}
$$

for all $\ell \geq 1$. We will need to consider three cases in order to prove (5.6) via (5.5), depending on where $\lambda_{0}$ falls relative to the support of $a$.

Case 1. $\left|\lambda_{0}\right| \lesssim 1,\left|\lambda_{0}\right| \gtrsim|\xi|^{-1}+\left|\xi^{\prime}\right|^{-1}$.

Note that the second inequality here implies that

$$
\frac{\xi+\left|\xi^{\prime}\right|}{t} \gtrsim \frac{\xi+\left|\xi^{\prime}\right|}{\xi\left|\xi^{\prime}\right|} \text { or } 1 \gtrsim \frac{t}{\xi\left|\xi^{\prime}\right|}
$$

Furthermore, we remark that $a \equiv 0$ unless $\xi \gtrsim 1$ and $\left|\xi^{\prime}\right| \gtrsim 1$. Starting with the first integral on the right-hand side of (5.5) we conclude from (5.10) that

$$
\delta^{2} \int \frac{\left|a_{\nu}\left(\lambda ; \xi, \xi^{\prime}\right)\right|}{\left|\lambda-\lambda_{0}\right|^{2}+\delta^{2}} d \lambda \lesssim\left(\langle\xi\rangle\left\langle\xi^{\prime}\right\rangle\right)^{-\frac{d}{2}-\sigma} t^{-1 / 2} \lesssim t^{-\frac{d+1}{2}-\sigma} .
$$

For the second integral in (5.5) we obtain from (5.11) that

$$
\delta^{2} \int_{\left|\lambda-\lambda_{0}\right|>\delta} \frac{\left|\partial_{\lambda} a_{\nu}\left(\lambda ; \xi, \xi^{\prime}\right)\right|}{\left|\lambda-\lambda_{0}\right|} d \lambda \lesssim\left(\langle\xi\rangle\left\langle\xi^{\prime}\right\rangle\right)^{-\frac{d}{2}-\sigma} t^{-\frac{1}{2}} \lesssim t^{-\frac{d+1}{2}-\sigma} .
$$

Case 2. $\left|\lambda_{0}\right| \lesssim 1,\left|\lambda_{0}\right| \ll\langle\xi\rangle^{-1}+\left\langle\xi^{\prime}\right\rangle^{-1}$.

In this case, we do not use Lemma 5.3. Instead we note that on the support of $a_{\nu}$, we have $\left|\partial_{\lambda} \phi\left(\lambda ; \xi, \xi^{\prime}\right)\right| \sim \lambda,\left|\partial_{\lambda}^{2} \phi\left(\lambda ; \xi, \xi^{\prime}\right)\right| \lesssim 1$ and the higher derivatives vanish. Let

$$
\lambda_{1}:=\max \left(\xi^{-1},\left|\xi^{\prime}\right|^{-1}\right) .
$$

Integrating by parts thus yields, for sufficiently large $N$,

$$
\begin{aligned}
& \left|\int e^{i t \phi\left(\lambda ; \xi, \xi^{\prime}\right)} a_{\nu}\left(\lambda ; \xi, \xi^{\prime}\right) d \lambda\right| \lesssim t^{-N} \int\left|\left(\partial_{\lambda}\left(\partial_{\lambda} \phi\right)^{-1}\right)^{N} a_{\nu}\left(\lambda ; \xi, \xi^{\prime}\right)\right| d \lambda \\
& \lesssim t^{-N} \int_{\lambda_{1}}^{1} \lambda^{2 \nu-2 N} d \lambda\left(\langle\xi\rangle\left\langle\xi^{\prime}\right\rangle\right)^{-\frac{d}{2}-\sigma} \\
& \lesssim t^{-N}\left(\min \left(\xi,\left|\xi^{\prime}\right|\right)\right)^{-2 \nu-1+2 N}\left(\langle\xi\rangle\left\langle\xi^{\prime}\right\rangle\right)^{-\frac{d}{2}-\sigma} \lesssim t^{-N}\left(\langle\xi\rangle\left\langle\xi^{\prime}\right\rangle\right)^{N-\frac{d+1}{2}-\sigma-\nu} \lesssim t^{-\frac{d+1}{2}-\sigma},
\end{aligned}
$$

where we used that $\xi\left|\xi^{\prime}\right| \lesssim t$, which follows from $\lambda_{0} \ll \lambda_{1}$.

Case 3. $\left|\lambda_{0}\right| \gg 1,\left|\lambda_{0}\right| \gtrsim \xi^{-1}+\left|\xi^{\prime}\right|^{-1}$.

In this case, $\left|\lambda-\lambda_{0}\right| \sim\left|\lambda_{0}\right| \gg 1$. Thus,

$$
\begin{aligned}
& \delta^{2} \int \frac{|a(\lambda)|}{\left|\lambda-\lambda_{0}\right|^{2}+t^{-1}} d \lambda \lesssim t^{-2}\left(\langle\xi\rangle\left\langle\xi^{\prime}\right\rangle\right)^{-\frac{d}{2}-\sigma} \lesssim t^{-\frac{d+4}{2}-\sigma}, \\
& \delta^{2} \int_{\left|\lambda-\lambda_{0}\right|>\delta} \frac{\left|a^{\prime}(\lambda)\right|}{\left|\lambda-\lambda_{0}\right|} d \lambda \lesssim t^{-1}\left(\langle\xi\rangle\left\langle\xi^{\prime}\right\rangle\right)^{-\frac{d}{2}-\sigma} \lesssim t^{-\frac{d+2}{2}-\sigma}
\end{aligned}
$$

and (5.6) is proved. 
For (5.7) note that by $N$-fold integration by parts,

$$
\left|\int_{-\infty}^{\infty} e^{i \lambda\left( \pm t+\xi-\xi^{\prime}\right)} a_{\nu}\left(\lambda ; \xi, \xi^{\prime}\right) d \lambda\right| \lesssim\left(1+\left|t+\xi-\xi^{\prime}\right|\right)^{-2 \nu-1}\left(\langle\xi\rangle\left\langle\xi^{\prime}\right\rangle\right)^{-\frac{d}{2}-\sigma} .
$$

However, since $2 \nu+1 \geq \nu+\frac{1}{2} \geq \frac{d}{2}+\sigma$, this expression is $\lesssim t^{-\frac{d}{2}-\sigma}$ uniformly in $\xi, \xi^{\prime}$ as claimed.

Now we turn to the estimate of the oscillatory integral for the case $|\xi \lambda|>1$ and $\left|\xi^{\prime} \lambda\right|<1$.

Lemma 5.5. Let $0 \leq \sigma \leq \nu-\frac{d-1}{2}$. For all $t>1$,

$$
\begin{gathered}
\sup _{\xi>0>\xi^{\prime}}\left|\left(\langle\xi\rangle\left\langle\xi^{\prime}\right\rangle\right)^{-\frac{d}{2}-\sigma} \int_{-\infty}^{\infty} e^{i t \lambda^{2}} \frac{\lambda \chi(\lambda)}{W_{\nu}(\lambda)} \chi_{\left[|\xi \lambda|>1,\left|\xi^{\prime} \lambda\right|<1\right]} f_{+, \nu}(\xi, \lambda) f_{-, \nu}\left(\xi^{\prime}, \lambda\right) d \lambda\right| \\
\lesssim t^{-\frac{d+1}{2}-\sigma}
\end{gathered}
$$

$$
\begin{aligned}
& \sup _{\xi>0>\xi^{\prime}}\left|\left(\langle\xi\rangle\left\langle\xi^{\prime}\right\rangle\right)^{-\frac{d}{2}-\sigma} \int_{-\infty}^{\infty} e^{ \pm i t \lambda} \frac{\lambda \chi(\lambda)}{W_{\nu}(\lambda)} \chi_{\left[|\xi \lambda|>1,\left|\xi^{\prime} \lambda\right|<1\right]} f_{+, \nu}(\xi, \lambda) f_{-, \nu}\left(\xi^{\prime}, \lambda\right) d \lambda\right| \\
& \lesssim t^{-\frac{d}{2}-\sigma}
\end{aligned}
$$

and similarly with $\chi_{\left[|\xi \lambda|<1,\left|\xi^{\prime} \lambda\right|>1\right]}$.

Proof. As before, we write $f_{+, \nu}(\xi, \lambda)=e^{i \xi \lambda} m_{+, \nu}(\xi, \lambda)$. But because of $\left|\xi^{\prime} \lambda\right|<1$ we use the representation

$$
f_{-, \nu}\left(\xi^{\prime}, \lambda\right)=a_{-, \nu}(\lambda) u_{0, \nu}^{-}\left(\xi^{\prime}, \lambda\right)+b_{-, \nu}(\lambda) u_{1, \nu}^{-}\left(\xi^{\prime}, \lambda\right) .
$$

In particular, Proposition 3.12, Lemma 3.2, and Corollary 3.5 yield

$$
\left|f_{-, \nu}\left(\xi^{\prime}, \lambda\right)\right| \lesssim|\lambda|^{\frac{1}{2}-\nu}\left\langle\xi^{\prime}\right\rangle^{\frac{1}{2}+\nu}, \quad\left|\partial_{\lambda} f_{-, \nu}\left(\xi^{\prime}, \lambda\right)\right| \lesssim|\lambda|^{-\frac{1}{2}-\nu}\left\langle\xi^{\prime}\right\rangle^{\frac{1}{2}+\nu}
$$

provided $\left|\xi^{\prime} \lambda\right|<1$. To obtain (5.15) we apply (5.5) with

$$
\phi(\lambda)=\phi\left(\lambda ; \xi, \xi^{\prime}\right)=\lambda^{2}+\frac{\xi}{t} \lambda
$$

and

$$
a(\lambda)=a_{\nu}\left(\lambda ; \xi, \xi^{\prime}\right)=\frac{\lambda \chi(\lambda)}{W_{\nu}(\lambda)}\left(\langle\xi\rangle\left\langle\xi^{\prime}\right\rangle\right)^{-\frac{d}{2}-\sigma} \chi_{\left[|\xi \lambda|>1,\left|\xi^{\prime} \lambda\right|<1\right]} m_{+, \nu}(\xi, \lambda) f_{-, \nu}\left(\xi^{\prime}, \lambda\right) .
$$

By Proposition 3.12 and (5.17),

$$
\begin{aligned}
|a(\lambda)| & \lesssim|\lambda|^{\frac{1}{2}+\nu}\langle\xi\rangle^{-\frac{d}{2}-\sigma}\left\langle\xi^{\prime}\right\rangle^{\nu-\sigma-\frac{d-1}{2}} \chi(\lambda) \chi_{\left[|\xi \lambda|>1,\left|\xi^{\prime} \lambda\right|<1\right]}, \\
\left|\partial_{\lambda}^{\ell} a(\lambda)\right| & \lesssim|\lambda|^{\frac{1}{2}+\nu-\ell}\langle\xi\rangle^{-\frac{d}{2}-\sigma}\left\langle\xi^{\prime}\right\rangle^{\nu-\sigma-\frac{d-1}{2}} \chi(\lambda) \chi_{\left[|\xi \lambda|>1,\left|\xi^{\prime} \lambda\right|<1\right]} \quad \forall \ell \geq 1 .
\end{aligned}
$$

The critical point of the phase is $\lambda_{0}=-\frac{\xi}{2 t}$. As usual, we begin with the true stationary phase case, i.e., $\lambda_{0} \in \operatorname{supp}(a)$. 
Case 1. $\left|\lambda_{0}\right| \lesssim 1,\left|\xi \lambda_{0}\right| \gtrsim 1,\left|\xi^{\prime} \lambda_{0}\right| \lesssim 1$

In this case, $\left|\xi \lambda_{0}\right| \gtrsim 1$ implies that $\xi \gtrsim t^{\frac{1}{2}}$, whereas $\left|\lambda_{0}\right| \lesssim 1$ and $\left|\xi^{\prime} \lambda_{0}\right| \lesssim 1$ together imply that $\langle\xi\rangle\left\langle\xi^{\prime}\right\rangle \lesssim t$. As a consequence, we remark that $\left|\lambda_{0}\right| \gtrsim \delta=t^{-\frac{1}{2}}$. Thus, letting $\chi_{\delta}$ denote a smooth cutoff to a neighborhood of size $c \delta$ where $c$ is some small positive constant, we conclude that

$$
\begin{aligned}
& \left|\int e^{i t \phi(\lambda)} a(\lambda) d \lambda\right| \lesssim \int_{\left|\lambda-\lambda_{0}\right|<c \delta}|\lambda|^{\frac{1}{2}+\nu} d \lambda\langle\xi\rangle^{-\frac{d}{2}-\sigma}\left\langle\xi^{\prime}\right\rangle^{\nu-\sigma-\frac{d-1}{2}} \\
& \quad+t^{-N} \int\left|\left(\partial_{\lambda} \frac{1}{\phi^{\prime}}\right)^{N}\left(1-\chi_{\delta}\right) a\right| d \lambda \\
& \lesssim\langle\xi\rangle^{-\frac{d}{2}-\sigma}\left\langle\xi^{\prime}\right\rangle^{\nu-\sigma-\frac{d-1}{2}}\left[\lambda_{0}^{\frac{1}{2}+\nu} \delta\right. \\
& \left.\quad+t^{-N} \int_{\left|\lambda-\lambda_{0}\right|>c \delta}\left(\left|\lambda-\lambda_{0}\right|^{-N} \lambda^{\frac{1}{2}+\nu-N}+\left|\lambda-\lambda_{0}\right|^{-2 N} \lambda^{\frac{1}{2}+\nu}\right) d \lambda\right] .
\end{aligned}
$$

Carrying out the integrations one checks that the entire right-hand side is $\lesssim$ $t^{-\frac{d+1}{2}-\sigma}$.

Case 2. $\left|\lambda_{0}\right| \lesssim 1,\left|\xi^{\prime} \lambda_{0}\right|>1$.

Then $\langle\xi\rangle\left\langle\xi^{\prime}\right\rangle>t$. Using Lemma 5.3 therefore yields

$$
\begin{aligned}
\delta^{2} \int \frac{|a(\lambda)|}{\left|\lambda-\lambda_{0}\right|^{2}+\delta^{2}} d \lambda & \lesssim \delta^{2} \int_{\xi^{-1}}^{\left\langle\xi^{\prime}\right\rangle^{-1}} \frac{\lambda^{\frac{1}{2}+\nu}\langle\xi\rangle^{-\frac{d}{2}-\sigma}\left\langle\xi^{\prime}\right\rangle^{\nu-\sigma-\frac{d-1}{2}}}{\left|\lambda-\lambda_{0}\right|^{2}+\delta^{2}} d \lambda \\
& \lesssim \delta\left\langle\xi^{\prime}\right\rangle^{-\frac{1}{2}-\nu}\langle\xi\rangle^{-\frac{d}{2}-\sigma}\left\langle\xi^{\prime}\right\rangle^{\nu-\sigma-\frac{d-1}{2}} \\
& \lesssim \delta\left(\langle\xi\rangle\left\langle\xi^{\prime}\right\rangle\right)^{-\frac{d}{2}-\sigma} \lesssim t^{-\frac{d+1}{2}-\sigma} .
\end{aligned}
$$

Similarly,

$$
\begin{aligned}
\delta^{2} \int_{\left|\lambda-\lambda_{0}\right|>\delta} \frac{\left|\partial_{\lambda} a(\lambda)\right|}{\left|\lambda-\lambda_{0}\right|} d \lambda & \lesssim \delta \int_{\xi^{-1}}^{\left\langle\xi^{\prime}\right\rangle^{-1}} \lambda^{-\frac{1}{2}+\nu}\langle\xi\rangle^{-\frac{d}{2}-\sigma}\left\langle\xi^{\prime}\right\rangle^{\nu-\sigma-\frac{d-1}{2}} d \lambda \\
& \lesssim \delta\left\langle\xi^{\prime}\right\rangle^{-\frac{1}{2}-\nu}\langle\xi\rangle^{-\frac{d}{2}-\sigma}\left\langle\xi^{\prime}\right\rangle^{\nu-\sigma-\frac{d-1}{2}} \lesssim t^{-\frac{d+1}{2}-\sigma}
\end{aligned}
$$

as before.

Case 3. $\left|\lambda_{0}\right| \lesssim 1,\left|\xi \lambda_{0}\right| \ll 1$.

In this case we integrate by parts without using Lemma [5.3. As in the previous lemma, we use that $\left|\phi^{\prime}(\lambda)\right| \sim \lambda$ on the support of $a$. In view of (5.18) and (5.19),

$$
\begin{aligned}
\left|\int_{-\infty}^{\infty} e^{i t \phi(\lambda)} a(\lambda) d \lambda\right| & \lesssim t^{-N} \int_{-\infty}^{\infty}\left|\left(\partial_{\lambda} \phi^{\prime}(\lambda)^{-1}\right)^{N} a(\lambda)\right| d \lambda \\
& \lesssim t^{-N}\langle\xi\rangle^{-\frac{d}{2}-\sigma}\left\langle\xi^{\prime}\right\rangle^{\nu-\sigma-\frac{d-1}{2}} \int_{\xi^{-1}}^{\infty} \lambda^{\frac{1}{2}+\nu-2 N} d \lambda \\
& \lesssim t^{-N} \xi^{2 N-1-d-2 \sigma} \lesssim t^{-\frac{d+1}{2}-\sigma},
\end{aligned}
$$

where we used that $\left|\xi^{\prime}\right| \leq \xi \lesssim t^{\frac{1}{2}}$. 
Case 4. $\left|\lambda_{0}\right| \gg 1$.

Here we use Lemma 5.3, In view of (5.18) and (5.19),

$$
\begin{gathered}
\delta^{2} \int \frac{|a(\lambda)|}{\left|\lambda-\lambda_{0}\right|^{2}+t^{-1}} d \lambda \lesssim t^{-2}\langle\xi\rangle^{-\frac{d}{2}-\sigma} \lesssim t^{-\frac{d+4}{2}-\sigma}, \\
\delta^{2} \int_{\left|\lambda-\lambda_{0}\right|>\delta} \frac{\left|a^{\prime}(\lambda)\right|}{\left|\lambda-\lambda_{0}\right|} d \lambda \lesssim t^{-1}\langle\xi\rangle^{-\frac{d}{2}-\sigma} \lesssim t^{-\frac{d+2}{2}-\sigma} .
\end{gathered}
$$

This proves (5.15).

For (5.16), note that

$$
\left|\int_{-\infty}^{\infty} e^{i \lambda\left( \pm t+\xi-\xi^{\prime}\right)} a_{\nu}\left(\lambda ; \xi, \xi^{\prime}\right) d \lambda\right| \lesssim\left(1+\left| \pm t+\xi-\xi^{\prime}\right|\right)^{-\nu-\frac{3}{2}}\langle\xi\rangle^{-\frac{d}{2}-\sigma}
$$

However, since $\nu+\frac{3}{2} \geq \nu+\frac{1}{2} \geq \frac{d}{2}+\sigma$, this expression is $\lesssim t^{-\frac{d}{2}-\sigma}$ uniformly in $\xi, \xi^{\prime}$ as claimed.

The other case $\chi_{\left[|\xi \lambda|<1, \xi^{\prime} \lambda<-1\right]}$ is treated in an analogous fashion.

The remaining cases for the small energy contributions are $\xi>\xi^{\prime}>|\lambda|^{-1}$ and $\xi^{\prime}<\xi<-|\lambda|^{-1}$. By symmetry it will suffice to treat the former case. As usual, we need to consider reflection and transmission coefficients; therefore we write

$$
f_{-, \nu}(\xi, \lambda)=\alpha_{-, \nu}(\lambda) f_{+, \nu}(\xi, \lambda)+\beta_{-, \nu}(\lambda) \overline{f_{+, \nu}(\xi, \lambda)} .
$$

Then, with $W_{\nu}(\lambda)=W\left(f_{-, \nu}(\cdot, \lambda), f_{+, \nu}(\cdot, \lambda)\right)$,

$$
W_{\nu}(\lambda)=-\beta_{-}(\lambda) W\left(f_{+, \nu}(\cdot, \lambda), \overline{f_{+, \nu}(\cdot, \lambda)}\right)=2 i \lambda \beta_{-, \nu}(\lambda)
$$

and

$$
\begin{aligned}
\widetilde{W}_{\nu}(\lambda) & :=W\left(f_{-, \nu}(\cdot, \lambda), \overline{f_{+, \nu}(\cdot, \lambda)}\right) \\
& =\alpha_{-, \nu}(\lambda) W\left(f_{+, \nu}(\cdot, \lambda), \overline{f_{+, \nu}(\cdot, \lambda)}\right)=-2 i \lambda \alpha_{-, \nu}(\lambda) .
\end{aligned}
$$

Therefore,

$$
\lambda \frac{\beta_{-, \nu}(\lambda)}{W_{\nu}(\lambda)}=-\frac{1}{2 i}, \quad \lambda \frac{\alpha_{-, \nu}(\lambda)}{W_{\nu}(\lambda)}=\frac{\widetilde{W}_{\nu}(\lambda)}{W_{\nu}(\lambda)}=\mathrm{const}+O\left(\lambda^{\varepsilon}\right)
$$

as can be seen from Proposition 3.12. The $O\left(\lambda^{\varepsilon}\right)$ term is complex-valued and behaves like a symbol.

Lemma 5.6. For any $t>1$ and $0 \leq \sigma \leq \nu-\frac{d-1}{2}$,

$$
\sup _{\xi>\xi^{\prime}>0}\left|\left(\langle\xi\rangle\left\langle\xi^{\prime}\right\rangle\right)^{-\frac{d}{2}-\sigma} \int e^{i t \lambda^{2}} \frac{\lambda \chi(\lambda)}{W_{\nu}(\lambda)} \chi_{\left[\left|\xi^{\prime} \lambda\right|>1\right]} f_{+, \nu}(\xi, \lambda) f_{-, \nu}\left(\xi^{\prime}, \lambda\right) d \lambda\right| \lesssim t^{-\frac{d+1}{2}-\sigma},
$$

$$
\sup _{\xi>\xi^{\prime}>0}\left|\left(\langle\xi\rangle\left\langle\xi^{\prime}\right\rangle\right)^{-\frac{d}{2}-\sigma} \int e^{ \pm i t \lambda} \frac{\lambda \chi(\lambda)}{W_{\nu}(\lambda)} \chi_{\left[\left|\xi^{\prime} \lambda\right|>1\right]} f_{+, \nu}(\xi, \lambda) f_{-, \nu}\left(\xi^{\prime}, \lambda\right) d \lambda\right| \lesssim t^{-\frac{d}{2}-\sigma}
$$

and similarly for $\sup _{\xi^{\prime}<\xi<0}$ and $\chi_{[|\xi \lambda|>1]}$. 
Proof. Using (5.21), we reduce (5.22) to two estimates, see (5.8):

$$
\begin{aligned}
& \sup _{\xi>\xi^{\prime}>0}\left(\langle\xi\rangle\left\langle\xi^{\prime}\right\rangle\right)^{-\frac{d}{2}-\sigma}\left|\int e^{i t \lambda^{2}} e^{i \lambda\left(\xi+\xi^{\prime}\right)} \chi_{\left[\xi^{\prime}|\lambda|>1\right]} \chi(\lambda) m_{+}(\xi, \lambda) m_{+}\left(\xi^{\prime}, \lambda\right) d \lambda\right| \\
& \lesssim t^{-\frac{d+1}{2}-\sigma} \\
& \sup _{\xi>\xi^{\prime}>0}\left(\langle\xi\rangle\left\langle\xi^{\prime}\right\rangle\right)^{-\frac{d}{2}-\sigma}\left|\int e^{i t \lambda^{2}} e^{i \lambda\left(\xi-\xi^{\prime}\right)} O(1) \chi_{\left[\xi^{\prime}|\lambda|>1\right]} \chi(\lambda) m_{+}(\xi, \lambda) \overline{m_{+}\left(\xi^{\prime}, \lambda\right)} d \lambda\right| \\
& \lesssim t^{-\frac{d+1}{2}-\sigma} \text {. }
\end{aligned}
$$

We apply (5.5) to (5.24) with fixed $\xi>\xi^{\prime}>0$ and

$$
\begin{aligned}
& \phi(\lambda):=\lambda^{2}+\frac{\lambda}{t}\left(\xi+\xi^{\prime}\right), \\
& a(\lambda):=\left(\langle\xi\rangle\left\langle\xi^{\prime}\right\rangle\right)^{-\frac{d}{2}-\sigma} \chi_{\left[\xi^{\prime}|\lambda|>1\right]} \chi(\lambda) m_{+}(\xi, \lambda) m_{+}\left(\xi^{\prime}, \lambda\right) .
\end{aligned}
$$

Then

$$
\begin{aligned}
|a(\lambda)| & \lesssim\left(\langle\xi\rangle\left\langle\xi^{\prime}\right\rangle\right)^{-\frac{d}{2}-\sigma} \chi(\lambda) \chi_{\left[\xi^{\prime}|\lambda|>1\right]}, \\
\left|\partial_{\lambda}^{\ell} a(\lambda)\right| & \lesssim|\lambda|^{-\ell}\left(\langle\xi\rangle\left\langle\xi^{\prime}\right\rangle\right)^{-\frac{d}{2}-\sigma} \chi(\lambda) \chi_{\left[\xi^{\prime}|\lambda|>1\right]} \quad \forall \ell \geq 1 .
\end{aligned}
$$

Case 1 . Suppose $\left|\lambda_{0}\right| \lesssim 1$ and $\left|\xi^{\prime} \lambda_{0}\right|>1$, where $\lambda_{0}=-\frac{\xi+\xi^{\prime}}{2 t}$. Note $\xi>\xi^{\prime} \gtrsim 1$.

Then

$$
\begin{aligned}
\delta^{2} \int \frac{|a(\lambda)|}{\left|\lambda-\lambda_{0}\right|^{2}+t^{-1}} d \lambda & \lesssim \delta^{2}\left(\langle\xi\rangle\left\langle\xi^{\prime}\right\rangle\right)^{-\frac{d}{2}-\sigma} \int \frac{d \lambda}{\left|\lambda-\lambda_{0}\right|^{2}+t^{-1}} \\
& \lesssim t^{-\frac{1}{2}} t^{-\frac{d}{2}-\sigma}
\end{aligned}
$$

since $\left|\xi^{\prime} \lambda_{0}\right| \sim \frac{\xi \xi^{\prime}}{t}>1$. As for the derivative term in (5.5), we infer from (5.27) that

$$
\delta^{2} \int_{\left|\lambda-\lambda_{0}\right|>\delta} \frac{\left|a^{\prime}(\lambda)\right|}{\left|\lambda-\lambda_{0}\right|} d \lambda \lesssim \delta^{2}\left(\langle\xi\rangle\left\langle\xi^{\prime}\right\rangle\right)^{-\frac{d}{2}-\sigma} \int_{\left|\lambda-\lambda_{0}\right|>\delta} \frac{d \lambda}{|\lambda|\left|\lambda-\lambda_{0}\right|} \chi_{\left[\left|\lambda \xi^{\prime}\right|>1\right]}
$$

We need to distinguish between $\left|\lambda-\lambda_{0}\right|>\frac{1}{10}\left|\lambda_{0}\right|$ and $\left|\lambda-\lambda_{0}\right|<\frac{1}{10}\left|\lambda_{0}\right|$. Thus,

$$
\begin{aligned}
\text { (5.28) } & \lesssim \delta^{2}\left(\langle\xi\rangle\left\langle\xi^{\prime}\right\rangle\right)^{-\frac{d}{2}-\sigma}\left[\int_{1 / \xi^{\prime}}^{\infty} \frac{d \lambda}{\lambda^{2}}+\left|\lambda_{0}\right|^{-1} \log \left(t^{1 / 2}\left|\lambda_{0}\right|\right)\right] \\
& \lesssim \delta^{2}\left(\langle\xi\rangle\left\langle\xi^{\prime}\right\rangle\right)^{-\frac{d}{2}-\sigma}\left[\xi^{\prime}+t \xi^{-1} \log \left(\xi t^{-\frac{1}{2}}\right)\right] \lesssim \delta^{2}\left(\langle\xi\rangle\left\langle\xi^{\prime}\right\rangle\right)^{-\frac{d}{2}-\sigma+\frac{1}{2}} \\
& \lesssim t^{-\frac{d+1}{2}-\sigma}
\end{aligned}
$$

where we used $\xi>\xi^{\prime} \gtrsim 1, \xi \xi^{\prime} \gtrsim t$, and $\xi^{2}>t$. 
Case $2 .\left|\lambda_{0}\right| \lesssim 1,\left|\lambda_{0}\right| \ll \frac{1}{\xi^{\prime}}$.

Then $\left|\phi^{\prime}(\lambda)\right| \sim|\lambda|$ on the support of $a(\lambda)$. Hence, integration by parts yields

$$
\begin{aligned}
& \left|\int e^{i t \phi(\lambda)} a(\lambda) d \lambda\right| \lesssim t^{-N} \int\left|\left(\partial_{\lambda} \phi^{\prime}(\lambda)^{-1}\right)^{N} a(\lambda)\right| d \lambda \\
& \lesssim t^{-N}\left(\langle\xi\rangle\left\langle\xi^{\prime}\right\rangle\right)^{-\frac{d}{2}-\sigma} \int_{\frac{1}{\xi^{\prime}}}^{\infty} \lambda^{-2 N} d \lambda \lesssim t^{-N}\left(\xi^{\prime}\right)^{2 N-d-1-2 \sigma} \lesssim t^{-\frac{d+1}{2}-\sigma},
\end{aligned}
$$

where we used that $\left(\xi^{\prime}\right)^{2}<\xi \xi^{\prime} \lesssim t$.

Case 3. $\left|\lambda_{0}\right| \gg 1,\left|\lambda_{0}\right| \gtrsim \frac{1}{\xi^{\prime}}$.

Then $\left|\lambda-\lambda_{0}\right| \sim\left|\lambda_{0}\right|$ on $\operatorname{supp}(a)$ and $\xi>t$. Using Lemma 5.3 yields

$$
\delta^{2} \int \frac{|a(\lambda)|}{\left|\lambda-\lambda_{0}\right|^{2}+t^{-1}} d \lambda \lesssim t^{-2}\left(\langle\xi\rangle\left\langle\xi^{\prime}\right\rangle\right)^{-\frac{d}{2}-\sigma} \lesssim t^{-\frac{d+4}{2}-\sigma}
$$

as well as

$$
\begin{aligned}
\delta^{2} \int_{\left|\lambda-\lambda_{0}\right|>\delta} \frac{\left|a^{\prime}(\lambda)\right|}{\left|\lambda-\lambda_{0}\right|} d \lambda & \lesssim \delta^{2}\left(\langle\xi\rangle\left\langle\xi^{\prime}\right\rangle\right)^{-\frac{d}{2}-\sigma}\left|\lambda_{0}\right|^{-1} \int_{\frac{1}{\left\langle\xi^{\prime}\right\rangle}}^{1} \frac{d \lambda}{|\lambda|} \\
& \lesssim \delta^{2}\left(\langle\xi\rangle\left\langle\xi^{\prime}\right\rangle\right)^{-\frac{d}{2}-\sigma} \frac{t}{\xi} \log \left\langle\xi^{\prime}\right\rangle \lesssim \delta^{2}\left(\langle\xi\rangle\left\langle\xi^{\prime}\right\rangle\right)^{-\frac{d}{2}-\sigma} t\langle\xi\rangle^{-\frac{1}{2}} \\
& \lesssim \delta\left(\langle\xi\rangle\left\langle\xi^{\prime}\right\rangle\right)^{-\frac{d}{2}-\sigma} \lesssim t^{-\frac{d+1}{2}-\sigma} .
\end{aligned}
$$

This concludes the proof of (5.24). For (5.25) we argue analogously, with $\lambda_{0}=$ $-\frac{\xi-\xi^{\prime}}{2 t}$. Case 1 above applies without major changes since we again have $\xi \xi^{\prime} \gtrsim t$. In Case 2, however, we cannot guarantee that $\xi \xi \lesssim t$ as before (since we could have $\xi=\xi^{\prime}$ and $\lambda_{0}=0$, say). However, if $\xi \xi^{\prime} \gtrsim t$, then the calculations of Case 1 yield the desired conclusion. Finally, Case 3 is the same and (5.25) is proved.

For (5.23) we first note the bound, obtained by repeated integration by parts using (5.27):

$$
\left|\int_{-\infty}^{\infty} e^{i \lambda\left( \pm t+\xi-\xi^{\prime}\right)} a_{\nu}\left(\lambda ; \xi, \xi^{\prime}\right) d \lambda\right| \lesssim\left(1+\left| \pm t+\xi-\xi^{\prime}\right|\right)^{-N}\left(\langle\xi\rangle\left\langle\xi^{\prime}\right\rangle\right)^{-\frac{d}{2}-\sigma}\left|\xi^{\prime}\right|^{N-1}
$$

valid for all integers $N \geq 1$. Now let us choose $N$ such that $N \geq \frac{d}{2}+\sigma \geq N-1$. Then this bound is dominated by $t^{-\frac{d}{2}-\sigma}$ uniformly in $\xi, \xi^{\prime}$ as claimed.

The case of $\xi^{\prime}<\xi<0,|\xi \lambda|>1$ is treated analogously.

We are done with the contributions of small $\lambda$ to our main oscillatory integral. To conclude the proof of Theorem 1.2 it suffices to prove the following statement about the contributions from "large" energies.

Lemma 5.7. For all $t>0$,

$$
\sup _{\xi>\xi^{\prime}}\left|\left(\langle\xi\rangle\left\langle\xi^{\prime}\right\rangle\right)^{-\frac{d}{2}-\sigma} \int_{-\infty}^{\infty} e^{i t \lambda^{2}} \frac{\lambda(1-\chi)(\lambda)}{W_{\nu}(\lambda)} f_{+, \nu}(\xi, \lambda) f_{-, \nu}\left(\xi^{\prime}, \lambda\right) d \lambda\right| \lesssim t^{-\frac{d+1}{2}-\sigma},
$$

where $\sigma \geq 0$ is arbitrary. 
Proof. We observed above, see (5.21), that $W_{\nu}(\lambda)=-2 i \lambda \beta_{-, \nu}(\lambda)$. Since $\left|\beta_{-, \nu}(\lambda)\right|$ $\geq 1$, this implies that $\left|W_{\nu}(\lambda)\right| \geq 2|\lambda|$. In particular, $W_{\nu}(\lambda) \neq 0$ for every $\lambda \neq 0$. We shall write

$$
f_{+, \nu}(\xi, \lambda)=e^{i \xi \lambda} m_{+, \nu}(\xi, \lambda), \quad f_{-, \nu}(\xi, \lambda)=e^{-i \xi \lambda} m_{-, \nu}(\xi, \lambda) .
$$

The functions $m_{ \pm, \nu}(\xi, \lambda)$ satisfy the Volterra equation

$$
m_{+, \nu}(\xi, \lambda)=1+\int_{\xi}^{\infty} \frac{1-e^{-2 i(\tilde{\xi}-\xi) \lambda}}{2 i \lambda} \widetilde{V}_{\nu}(\tilde{\xi}) m_{+, \nu}(\tilde{\xi}, \lambda) d \tilde{\xi},
$$

where the potential $\widetilde{V}_{\nu}$ satisfies

$$
\left|\frac{d^{\ell}}{d \xi^{\ell}} \widetilde{V}_{\nu}(\xi)\right| \lesssim\langle\xi\rangle^{-2-\ell}, \quad \forall \ell \geq 0 .
$$

From (5.30), for any $\xi \geq 0$,

$$
m_{+, \nu}(\xi, \lambda)=1+O\left(\lambda^{-1}\langle\xi\rangle^{-1}\right) .
$$

Moreover, see [21],

$$
\left|\partial_{\lambda}^{k} \partial_{\xi}^{\ell} m_{+, \nu}(\xi, \lambda)\right| \leq C_{k, \ell} \lambda^{-1-k}\langle\xi\rangle^{-1-\ell} \quad \forall k+\ell>0 .
$$

In 21] this is proved for $k+\ell \leq 2$, but the proof there extends inductively to higher orders. As a corollary, we obtain (take $\xi=0$ )

$$
\begin{aligned}
W_{\nu}(\lambda)= & W\left(f_{-, \nu}(\cdot, \lambda), f_{+, \nu}(\cdot, \lambda)\right) \\
= & m_{+, \nu}(\xi, \lambda)\left[m_{-, \nu}^{\prime}(\xi, \lambda)-i \lambda m_{-, \nu}(\xi, \lambda)\right] \\
& -m_{-, \nu}(\xi, \lambda)\left[m_{+, \nu}^{\prime}(\xi, \lambda)+i \lambda m_{+, \nu}(\xi, \lambda)\right] \\
= & -2 i \lambda\left(1+O\left(\lambda^{-1}\right)\right)+O\left(\lambda^{-1}\right)=-2 i \lambda+O(1)
\end{aligned}
$$

with derivatives $\left(\lambda / W_{\nu}(\lambda)\right)^{(\ell)}=O\left(\lambda^{-1-\ell}\right)$ as $|\lambda| \rightarrow \infty$.

In order to prove (5.29), we will need to distinguish the cases $\xi>0>\xi^{\prime}$, $\xi>\xi^{\prime}>0$, as well as $0>\xi>\xi^{\prime}$. By symmetry, it will suffice to consider the first two.

Case 1. $\xi>0>\xi^{\prime}$.

In this case we need to prove that

$$
\begin{array}{r}
\sup _{\xi>0>\xi^{\prime}}\left|\left(\langle\xi\rangle\left\langle\xi^{\prime}\right\rangle\right)^{-\frac{d}{2}-\sigma} \int e^{i t\left[\lambda^{2}+\frac{\xi-\xi^{\prime}}{t} \lambda\right]} \frac{\lambda(1-\chi)(\lambda)}{W_{\nu}(\lambda)} m_{+, \nu}(\xi, \lambda) m_{-, \nu}\left(\xi^{\prime}, \lambda\right) d \lambda\right| \\
\lesssim t^{-\frac{d+1}{2}-\sigma} .
\end{array}
$$

Let $\phi(\lambda):=\lambda^{2}+\frac{\xi-\xi^{\prime}}{t} \lambda$ and

$$
a(\lambda):=\left(\langle\xi\rangle\left\langle\xi^{\prime}\right\rangle\right)^{-\frac{d}{2}-\sigma} \frac{\lambda(1-\chi)(\lambda)}{W_{\nu}(\lambda)} m_{+, \nu}(\xi, \lambda) m_{-, \nu}\left(\xi^{\prime}, \lambda\right) .
$$

The phase $\phi$ has critical point $\lambda_{0}=-\frac{\xi-\xi^{\prime}}{2 t}$. If $\left|\lambda_{0}\right| \ll 1$, then $\left|\phi^{\prime}(\lambda)\right| \sim|\lambda|$ on the support of $a$. Therefore, integrating by parts repeatedly yields that the oscillatory integral in (5.31) decays like $t^{-N}$ for all $N \geq 1$. It is important to note that already a single derivative in $\lambda$ renders $a(\lambda)$ of size $O\left(\lambda^{-2}\right)$ and thus integrable. 
For the remainder of Case 1 we can therefore assume that $\left|\lambda_{0}\right| \gtrsim 1$, which implies that $\max \left(\xi,\left|\xi^{\prime}\right|\right) \gtrsim t$. In particular, the factor $\left(\langle\xi\rangle\left\langle\xi^{\prime}\right\rangle\right)^{-\frac{d}{2}-\sigma}$ contributes the decay $t^{-\frac{d}{2}-\sigma}$. Applying Lemma 5.3 yields

$$
\begin{aligned}
\text { (5.31) } & \lesssim \delta^{2} \int \frac{|a(\lambda)|}{\left|\lambda-\lambda_{0}\right|^{2}+t^{-1}} d \lambda+\delta^{2} \int_{\left|\lambda-\lambda_{0}\right|>\delta} \frac{\left|a^{\prime}(\lambda)\right|}{\left|\lambda-\lambda_{0}\right|} d \lambda \\
& =: A+B .
\end{aligned}
$$

By the preceding,

$$
A \lesssim \delta\|a\|_{\infty} \lesssim t^{-\frac{1}{2}}\left(\langle\xi\rangle\left\langle\xi^{\prime}\right\rangle\right)^{-\frac{d}{2}-\sigma} \lesssim t^{-\frac{d+1}{2}-\sigma} .
$$

Next, we estimate $B$. First, from our bounds on $W_{\nu}(\lambda), m_{+, \nu}(\xi, \lambda)$ and $m_{-, \nu}\left(\xi^{\prime}, \lambda\right)$ we conclude that

$$
\left|a^{\prime}(\lambda)\right| \lesssim\left(\langle\xi\rangle\left\langle\xi^{\prime}\right\rangle\right)^{-\frac{d}{2}-\sigma} \chi_{[|\lambda| \gtrsim 1]}|\lambda|^{-2}
$$

Second, because of $\left|\lambda_{0}\right| \gtrsim 1$ we obtain

$$
\begin{aligned}
B & \lesssim \delta^{2}\left(\langle\xi\rangle\left\langle\xi^{\prime}\right\rangle\right)^{-\frac{d}{2}-\sigma} \int_{\substack{\left(\lambda \lambda \lambda_{0}|>\delta\\
| \lambda \mid \gtrsim 1\right.}} \frac{d \lambda}{|\lambda|^{2}\left|\lambda-\lambda_{0}\right|} \\
& \lesssim \delta\left(\langle\xi\rangle\left\langle\xi^{\prime}\right\rangle\right)^{-\frac{d}{2}-\sigma} \int_{|\lambda| \gtrsim 1} \frac{d \lambda}{\lambda^{2}} \lesssim t^{-\frac{d+1}{2}-\sigma} .
\end{aligned}
$$

This finishes the case $\xi>0>\xi^{\prime}$.

Case 2. $\xi>\xi^{\prime}>0$.

From (5.21),

$$
f_{-, \nu}\left(\xi^{\prime}, \lambda\right)=\alpha_{-, \nu}(\lambda) f_{+, \nu}\left(\xi^{\prime}, \lambda\right)+\beta_{-, \nu}(\lambda) \overline{f_{+, \nu}\left(\xi^{\prime}, \lambda\right)},
$$

where

$$
\begin{aligned}
& \alpha_{-, \nu}(\lambda)=\frac{W\left(f_{-, \nu}(\cdot, \lambda), \overline{f_{+, \nu}(\cdot, \lambda)}\right)}{-2 i \lambda}, \\
& \beta_{-, \nu}(\lambda)=\frac{W\left(f_{+, \nu}(\cdot, \lambda), f_{-, \nu}(\cdot, \lambda)\right)}{-2 i \lambda}=\frac{W_{\nu}(\lambda)}{2 i \lambda} .
\end{aligned}
$$

From our large $\lambda$ asymptotics of $W_{\nu}(\lambda)$ we deduce that

$$
\beta_{-, \nu}(\lambda)=1+O\left(\lambda^{-1}\right), \quad \beta_{-, \nu}^{\prime}(\lambda)=O\left(\lambda^{-2}\right) .
$$

For $\alpha_{-, \nu}(\lambda)$ we calculate, again at $\xi=0$,

$$
\begin{aligned}
W\left(f_{-, \nu}(\cdot, \lambda), \overline{f_{+, \nu}(\cdot, \lambda)}\right)= & m_{-, \nu}(\xi, \lambda)\left(\bar{m}_{+, \nu}^{\prime}(\xi, \lambda)-2 i \lambda \bar{m}_{+, \nu}(\xi, \lambda)\right) \\
& -\bar{m}_{+, \nu}(\xi, \lambda)\left(m_{-, \nu}^{\prime}(\xi, \lambda)-2 i \lambda m_{-, \nu}(\xi, \lambda)\right) \\
= & m_{-, \nu}(\xi, \lambda) \bar{m}_{+, \nu}^{\prime}(\xi, \lambda)-m_{-, \nu}^{\prime}(\xi, \lambda) \bar{m}_{+, \nu}(\xi, \lambda) \\
= & O\left(\lambda^{-1}\right)
\end{aligned}
$$

so that

$$
\alpha_{-, \nu}(\lambda)=O\left(\lambda^{-2}\right), \quad \alpha_{-, \nu}^{\prime}(\lambda)=O\left(\lambda^{-3}\right) .
$$


Thus, we are left with proving the two bounds

$\sup _{\xi>\xi^{\prime}>0}\left|\int_{-\infty}^{\infty} e^{i t \lambda^{2}} e^{i \lambda\left(\xi+\xi^{\prime}\right)} \frac{\lambda(1-\chi(\lambda))}{W_{\nu}(\lambda)} \alpha_{-, \nu}(\lambda) \frac{m_{+, \nu}(\xi, \lambda) m_{+, \nu}\left(\xi^{\prime}, \lambda\right)}{\left(\langle\xi\rangle\left\langle\xi^{\prime}\right\rangle\right)^{\frac{d}{2}+\sigma}} d \lambda\right| \lesssim t^{-\frac{d+1}{2}-\sigma}$,

$\sup _{\xi>\xi^{\prime}>0}\left|\int_{-\infty}^{\infty} e^{i t \lambda^{2}} e^{i \lambda\left(\xi-\xi^{\prime}\right)} \frac{\lambda(1-\chi(\lambda))}{W_{\nu}(\lambda)} \beta_{-, \nu}(\lambda) \frac{m_{+, \nu}(\xi, \lambda) \overline{m_{+, \nu}\left(\xi^{\prime}, \lambda\right)}}{\left(\langle\xi\rangle\left\langle\xi^{\prime}\right\rangle\right)^{\frac{d}{2}+\sigma}} d \lambda\right| \lesssim t^{-\frac{d+1}{2}-\sigma}$ for any $t>0$. This, however, follows by means of the exact same arguments which we use to prove (5.31). Note that in (5.34) the critical point of the phase is

$$
\lambda_{0}=-\frac{\xi+\xi^{\prime}}{2 t}
$$

whereas in (5.35) it is $\lambda_{0}=-\frac{\xi-\xi^{\prime}}{2 t}$. In either case it follows from $\left|\lambda_{0}\right| \gtrsim 1$ that $\xi \gtrsim t$. Hence we can indeed argue as in Case 1 . This finishes the proof of the lemma, and thus also establishes Theorem 1.2 .

Now for the wave case. We will tacitly use some elements of the previous proof.

Lemma 5.8. For all $t>0$,

$$
\begin{aligned}
& \left|\int_{-\infty}^{\xi}\left(\langle\xi\rangle\left\langle\xi^{\prime}\right\rangle\right)^{-\frac{d}{2}-\sigma} \int_{-\infty}^{\infty} e^{ \pm i t \lambda} \frac{\lambda(1-\chi)(\lambda)}{W_{\nu}(\lambda)} f_{+, \nu}(\xi, \lambda) f_{-, \nu}\left(\xi^{\prime}, \lambda\right) d \lambda \phi\left(\xi^{\prime}\right) d \xi^{\prime}\right| \\
& \lesssim t^{-\frac{d}{2}-\sigma} \int\left(|\phi(\eta)|+\left|\phi^{\prime}(\eta)\right|\right) d \eta
\end{aligned}
$$

with a constant that does not depend on $\xi$.

Proof. In order to prove (5.36), we will need to distinguish the cases $\xi>0>\xi^{\prime}$, $\xi>\xi^{\prime}>0$, and $0>\xi>\xi^{\prime}$. By symmetry, it will suffice to consider the first two.

Case 1. $\xi>0>\xi^{\prime}$.

Integrating by parts yields

$$
\begin{aligned}
& \left|\left(\langle\xi\rangle\left\langle\xi^{\prime}\right\rangle\right)^{-\frac{d}{2}-\sigma} \int e^{i \lambda\left( \pm t+\xi-\xi^{\prime}\right)} \frac{\lambda(1-\chi)(\lambda)}{W_{\nu}(\lambda)} m_{+, \nu}(\xi, \lambda) m_{-, \nu}\left(\xi^{\prime}, \lambda\right) d \lambda\right| \\
& \lesssim\left(\langle\xi\rangle\left\langle\xi^{\prime}\right\rangle\right)^{-\frac{d}{2}-\sigma}\left|t \pm\left(\xi-\xi^{\prime}\right)\right|^{-N} \lesssim t^{-\frac{d}{2}-\sigma}
\end{aligned}
$$

provided $\left|t \pm\left(\xi-\xi^{\prime}\right)\right| \geq 1$. If this fails, then we need to integrate by parts in $\xi^{\prime}$ to remove one factor of $\lambda$ : since $\lambda e^{-i \xi^{\prime} \lambda}=i \partial_{\xi^{\prime}} e^{-i \xi^{\prime} \lambda}$, it follows that

$$
\begin{aligned}
& \int_{-\infty}^{\xi}\langle\xi\rangle^{-\frac{d}{2}-\sigma}\left\langle\xi^{\prime}\right\rangle^{-\frac{d}{2}-\sigma} \int e^{i \lambda\left( \pm t+\xi-\xi^{\prime}\right)} \frac{\lambda(1-\chi)(\lambda)}{W_{\nu}(\lambda)} m_{+, \nu}(\xi, \lambda) m_{-, \nu}\left(\xi^{\prime}, \lambda\right) d \lambda \phi\left(\xi^{\prime}\right) d \xi^{\prime} \\
& =i\langle\xi\rangle^{-d-2 \sigma} \int e^{ \pm i t \lambda} \frac{(1-\chi)(\lambda)}{W_{\nu}(\lambda)} m_{+, \nu}(\xi, \lambda) m_{-, \nu}(\xi, \lambda) d \lambda \phi(\xi)-i \int_{-\infty}^{\xi}\langle\xi\rangle^{-\frac{d}{2}-\sigma} \\
& \times \int e^{i \lambda\left( \pm t+\xi-\xi^{\prime}\right)} \frac{(1-\chi)(\lambda)}{W_{\nu}(\lambda)} m_{+, \nu}(\xi, \lambda) \partial_{\xi^{\prime}}\left[\left\langle\xi^{\prime}\right\rangle^{-\frac{d}{2}-\sigma} m_{-, \nu}\left(\xi^{\prime}, \lambda\right) \phi\left(\xi^{\prime}\right)\right] d \lambda d \xi^{\prime}
\end{aligned}
$$

Denote the two expressions after the equality sign by $A$ and $B$, respectively. First, exploiting the cancellation due to $W_{\nu}(-\lambda)=-W_{\nu}(\lambda)+O(1)$ as $\lambda \rightarrow \infty$, we see that

$$
\sup _{\xi>0>\xi^{\prime}}\left|\int e^{i t \lambda} \frac{(1-\chi)(\lambda)}{W_{\nu}(\lambda)} m_{+, \nu}(\xi, \lambda) m_{-, \nu}(\xi, \lambda) d \lambda\right| \lesssim 1 .
$$


Furthermore, integrating by parts in $\lambda$ shows that the left-hand side is in fact $\lesssim t^{-N}$ for any $N$. Hence,

$$
A \lesssim\langle t\rangle^{-N} \sup |\phi| \leq\langle t\rangle^{-N} \int\left(\left|\phi^{\prime}(\xi)\right|+|\phi(\xi)|\right) d \xi .
$$

Second, by the same cancellation,

$$
\begin{aligned}
B & \lesssim \int\left(\langle\xi\rangle\left\langle\xi^{\prime}\right\rangle\right)^{-\frac{d}{2}-\sigma}\left(1+\left|t \pm\left(\xi-\xi^{\prime}\right)\right|\right)^{-N}\left(\left|\phi^{\prime}\left(\xi^{\prime}\right)\right|+\left|\phi\left(\xi^{\prime}\right)\right|\right) d \xi^{\prime} \\
& \lesssim t^{-\frac{d}{2}-\sigma} \int\left(\left|\phi^{\prime}\left(\xi^{\prime}\right)\right|+\left|\phi\left(\xi^{\prime}\right)\right|\right) d \xi^{\prime},
\end{aligned}
$$

which gives the desired bound as usual.

Case 2. $\xi>\xi^{\prime}>0$.

In analogy with (5.34) and (5.35) we need to consider

$$
\begin{aligned}
& \int_{-\infty}^{\infty} e^{i t \lambda} e^{i \lambda\left(\xi+\xi^{\prime}\right)} \frac{\lambda(1-\chi(\lambda))}{W_{\nu}(\lambda)} \alpha_{-, \nu}(\lambda) \frac{m_{+, \nu}(\xi, \lambda) m_{+, \nu}\left(\xi^{\prime}, \lambda\right)}{\left(\langle\xi\rangle\left\langle\xi^{\prime}\right\rangle\right)^{\frac{d}{2}+\sigma}} d \lambda, \\
& \int_{-\infty}^{\infty} e^{i t \lambda} e^{i \lambda\left(\xi-\xi^{\prime}\right)} \frac{\lambda(1-\chi(\lambda))}{W_{\nu}(\lambda)} \beta_{-, \nu}(\lambda) \frac{m_{+, \nu}(\xi, \lambda) \overline{m_{+, \nu}\left(\xi^{\prime}, \lambda\right)}}{\left(\langle\xi\rangle\left\langle\xi^{\prime}\right\rangle\right)^{\frac{d}{2}+\sigma}} d \lambda .
\end{aligned}
$$

The integral in (5.37) is $\lesssim\langle t\rangle^{-\frac{d}{2}-\sigma}$ uniformly in $\xi, \xi^{\prime}$ due to the decay of $\alpha_{-, \nu}$; see (5.33). On the other hand, the integral in (5.38) is not a bounded function in $\xi, \xi^{\prime}$ due to the lack of decay in $\lambda$; see (5.32). Thus, we again need to redeem one power of $\lambda$ via a $\xi^{\prime}$ differentiation; see above.

\section{REFERENCES}

1. M. Abramowitz \& I. Stegun, Handbook of mathematical functions with formulas, graphs, and mathematical tables, Reprint of the 1972 edition. Wiley-Interscience Publication; National Bureau of Standards, Washington, DC, 1984. MR757537 (85j:00005a)

2. J. Bourgain, Fourier transform restriction phenomena for certain lattice subsets and applications to nonlinear evolution equations. I. Schrödinger equations, Geom. Funct. Anal. 3 (1993), 107-156. MR1209299 (95d:35160a)

3. N. Burq, Décroissance de l'énergie locale de l'équation des ondes pour le problème extérieur et absence de résonance au voisinage du réel. Acta Math. 180 (1998), no. 1, 1-29. MR.1618254 (99j:35119)

4. N. Burq, P. Gérard \& N. Tzvetkov, Strichartz inequalities and the nonlinear Schrödinger equation on compact manifolds, Amer. J. Math. 126 (2004), 569-605. MR2058384 (2005h:58036)

5. N. Burq, P. Gérard \& N. Tzvetkov, Bilinear eigenfunction estimates and the nonlinear Schrödinger equation on surfaces, Invent. Math. 159 (2005), 187-223. MR2142336 (2005m:35275)

6. H. Christianson, Semiclassical non-concentration near hyperbolic orbits. J. Funct. Anal. 246 (2007), 145-195. MR2321040

7. H. Christianson, Dispersive estimates for manifolds with one trapped orbit, preprint 2007.

8. H. Christianson, Cutoff resolvent estimates and the semilinear Schrödinger equation, Proc. Amer. Math. Soc. 136 (2008), 3513-3520. MR.2415035

9. W. Craig, T. Kappeler \& W. Strauss, Microlocal dispersive smoothing for the Schrödinger equation, Comm. Pure Appl. Math. 48 (1995), 769-860. MR1361016 (96m:35057)

10. P. Deift \& E. Trubowitz, Inverse scattering on the line, Comm. Pure Appl. Math. XXXII (1979), 121-251. MR.512420 (80e:34011)

11. S. Doi, Smoothing effects for Schrödinger evolution equation and global behavior of geodesic flow, Math. Ann. 318 (2000), 355-389. MR.1795567 (2001h:58045)

12. C. Gérard \& J. Sjöstrand, Semiclassical resonances generated by a closed trajectory of hyperbolic type. Comm. Math. Phys. 108 (1987), no. 3, 391-421. MR874901 (88k:58151) 
13. P. Gérard, Nonlinear Schrödinger equations on compact manifolds. European Congress of Mathematics, Eur. Math. Soc. Zürich, 121-139, 2005. MR2185741 (2006g:58057)

14. A. Hassell, T. Tao \& J. Wunsch, A Strichartz inequality for the Schrödinger equation on non-trapping asymptotically conic manifolds, Commun. Partial Differ. Equations 30 (2005), 157-205. MR2131050 (2006i:58045)

15. A. Hassell, T. Tao \& J. Wunsch, Sharp Strichartz estimates on non-trapping asymptotically conic manifolds, American Journal of Mathematics, 128 (2006), 963-1024. MR2251591 (2007d:58053)

16. S. Nonnenmacher \& M. Zworski, Quantum decay rates in chaotic scattering, Équations aux Dérivées Partielles. 2005-2006, Exp. No. XXII, 8 pp., Sémin. Équ. Dériv. Partielles, École Polytech., Palaiseau, 2006. MR2276087(2007i:35177)

17. L. Robbiano \& C. Zuily, Strichartz estimates for Schrödinger equations with variable coeffcients, Mém. Soc. Math. Fr. (N.S.) No. 101-102, 2005. MR2193021(2006i:35047)

18. I. Rodnianski \& T. Tao, Longtime decay estimates for the Schrödinger equation on manifolds. Mathematical aspects of nonlinear dispersive equations, 223-253, Ann. of Math. Stud., 163, Princeton Univ. Press, Princeton, NJ, 2007. MR2333213 (2008g:58035)

19. W. Schlag, Dispersive estimates for Schrödinger operators in dimension two, Comm. Math. Phys. 257 (2005), 87-117. MR2163570 (2006d:35045)

20. W. Schlag, Dispersive estimates for Schrödinger operators: A survey, Proceedings of the conference "Workshop on Aspects of nonlinear PDE's", Ann. of Math. Stud. 163, Princeton, NJ, 2007. MR2333215

21. W. Schlag, A. Soffer \& W. Staubach, Decay for the wave and Schrödinger evolutions on manifolds with conical ends, Part I, to appear in Trans. Amer. Math. Soc.

22. H. Smith \& C. Sogge, Global Strichartz estimates for nontrapping perturbations of the Laplacian, Commun. Partial Differ. Equations 25 (2000), 2171-2183. MR.1789924 (2001j:35180)

23. G. Staffilani \& D. Tataru, Strichartz estimates for a Schrödinger operator with nonsmooth coefficients, Commun. Partial Differ. Equations 27 (2002), 1337-1372. MR.1924470 (2003f:35248)

24. D. Tataru, Parametrices and dispersive estimates for Schrödinger operators with variable coefficients, Amer. J. Math. 130 (2008), 571-634. MR2418923

Department of Mathematics, University of Chicago, 5734 South University Avenue, Chicago, Illinois 60637

E-mail address: schlag@math.uchicago.edu

Department of Mathematics, Rutgers University, 110 Freylinghuysen Road, PiscatAWAY, NEW JERSEY 08854

E-mail address: soffer@math.rutgers.edu

Department of Mathematics, Colin Maclaurin Bullding, Heriot-Watt University, EDinburgh, EH14 4AS, Scotland

E-mail address: W.Staubach@hw.ac.uk 